\title{
The h Channel Mediates Location Dependence and Plasticity of Intrinsic Phase Response in Rat Hippocampal Neurons
}

\author{
Rishikesh Narayanan and Daniel Johnston \\ Center for Learning and Memory, The University of Texas at Austin, Austin, Texas 78712
}

\begin{abstract}
The presence of phenomenological inductances in neuronal membrane has been known for more than one-half a century. Despite this, the dramatic contributions of such inductive elements to the amplitude and, especially, phase of neuronal impedance, and their roles in modulating temporal dynamics of neuronal responses have surprisingly remained unexplored. In this study, we demonstrate that the $h$ channel contributes a location-dependent and plastic phenomenological inductive component to the input impedance of CA1 pyramidal neurons. Specifically, we show that the $h$ channels introduce an apparent negative delay in the local voltage response of these neurons with respect to the injected current within the theta frequency range. The frequency range and the extent of this lead expand with increases in $\mathrm{h}$ current either through hyperpolarization, or with increasing distance of dendritic location from the soma. We also demonstrate that a spatially widespread increase in this inductive phase component accompanies long-term potentiation. Finally, using impedance analysis, we show that both location and activity dependence of intrinsic phase response are attributable not to changes in a capacitive or a leak component, but to changes in $\mathrm{h}$-channel properties. Our results suggest that certain voltage-gated ion channels can differentially regulate internal time delays within neurons, thus providing them with an independent control mechanism in temporal coding of neuronal information. Our analyses and results also establish impedance as a powerful measure of intrinsic dynamics and excitability, given that it quantifies temporal relationships among signals and excitability as functions of input frequency.
\end{abstract}

Key words: activity-dependent plasticity; dendrite; h channel; hippocampus; impedance phase; inductance; temporal coding

\section{Introduction}

Electrical impedance, the ratio of the voltage response to the injected current, is a standard measurement for characterizing neuronal membrane properties. Impedance is a complex number with resistance forming its real part and reactance its imaginary part. Whereas resistance is a positive quantity, reactance can be either positive or negative, depending on the presence of inductive or capacitive elements, respectively. A reactance of zero implies that the current and voltage are in phase, whereas a positive or a negative reactance indicates that the voltage response leads or lags the input current, respectively (Middendorf, 1965; Skilling, 1965).

The first measurements of inductive reactance in neuronal membrane were made in the squid giant axon (Cole and Baker, 1941). It was later demonstrated that these inductive reactances as well as their capacitive counterparts emerge because of the presence of voltage-dependent, time-variant conductances (Mauro, 1961; Cole, 1968; Sabah and Leibovic, 1969; Mauro et al., 1970), and such reactances have been variously named as "anomalous" (Cole, 1949) or "phenomenological" reactances

Received Feb. 25, 2008; revised April 25, 2008; accepted April 28, 2008.

This work was supported by the International Human Frontier Science Program Organization (R.N.) and National Institutes of Health Grants MH48432, MH44754, and NS37444 (D.J.). We thank Drs. Jeffrey Magee and Xixi Chen and members of the Johnston Laboratory for helpful comments on a draft of this manuscript.

Correspondence should be addressed to Dr. Daniel Johnston, Center for Learning and Memory, The University of Texas at Austin, 1, University Station Stop, C7000, Austin, TX 78712-0805. E-mail: djohnston@mail.clm.utexas.edu. DOI:10.1523/JNEUROSCI.0835-08.2008

Copyright $\odot 2008$ Society for Neuroscience $\quad$ 0270-6474/08/285846-15\$15.00/0
(Mauro et al., 1970; Koch, 1984). Importantly, whereas excitatory conductance changes contribute to a capacitive reactance, conductance changes that aid in membrane recovery yield an inductive reactance (Cole, 1968; Sabah and Leibovic, 1969).

Electrical resonance has been reported in multiple cell types and is mediated by conductances designated as resonating conductances (Hutcheon and Yarom, 2000), all of which could be considered as phenomenological inductances. Although there have been multiple studies on impedance amplitude and associated resonance properties (Puil et al., 1986; Llinas, 1988; Gutfreund et al., 1995; Hutcheon et al., 1996a; Leung and Yu, 1998; Hutcheon and Yarom, 2000; Pike et al., 2000; Hu et al., 2002; Schreiber et al., 2004; Narayanan and Johnston, 2007), impedance phase has not been quantitatively assessed. Given that in vivo electrophysiologists recognize phase as a critical determinant of neuronal function (O'Keefe and Recce, 1993; Kamondi et al., 1998; Buzsaki, 2002; Mehta et al., 2002; Hasselmo, 2005; Lengyel et al., 2005; Lisman, 2005; O'Keefe and Burgess, 2005; Siapas et al., 2005; Fries et al., 2007), this lacuna in in vitro analysis of impedance phase is striking. An aim of this study is to fill this lacuna through the use of quantitative techniques derived and developed from methodologies used in analyzing electrical circuits (Middendorf, 1965; Skilling, 1965).

The $\mathrm{h}$ conductance is a resonating conductance (Hutcheon et al., 1996b), and neurons that express it have been modeled as equivalent RCL circuits (Demontis et al., 1999; Hutcheon and Yarom, 2000; Armstrong-Gold and Rieke, 2003; Mao et al., 2003; Erchova et al., 2004). In a recent study involving the h conduc- 
tance, we had established the location and activity dependence of the resonance frequency of CA1 pyramidal neurons (Narayanan and Johnston, 2007). In this study, we quantitatively analyze impedance phase and demonstrate that the h channel contributes a location-dependent and plastic inductive component to the input impedance of these neurons. Specifically, we demonstrate that the $h$ channel intrinsically provides apparent negative time delays to theta frequency inputs arriving into a neuron. Consistent with the reported gradient of h-channel density along the somatoapical axis (Magee, 1998; Lorincz et al., 2002), this lead in input impedance phase increases as a function of distance from the soma. Finally, we show that long-term potentiation is accompanied by a spatially widespread increase in this inductive phase component, attained through activity-dependent modulation of h-channel properties. Our results suggest that phenomenological inductances can differentially regulate internal time delays within neurons, thus providing them with an independent control mechanism in temporal coding of neuronal information.

\section{Materials and Methods}

Surgery and slice preparation. All experiments were conducted in accordance with the rules and regulations of The University of Texas at Austin Institutional Animal Care and Use Committee. Male Sprague Dawley rats (5-7 weeks of age) were anesthetized through intraperitoneal injection of a combination of ketamine and xylazine. After the rat ceased to respond to foot pinch, it was transcardially perfused with ice-cold $\left(4^{\circ} \mathrm{C}\right)$ cutting solution (in mм: 210 sucrose, $2.5 \mathrm{KCl}, 1.25 \mathrm{NaH}_{2} \mathrm{PO}_{4}, 25$ $\mathrm{NaHCO}_{3}, 0.5 \mathrm{CaCl}_{2}, 7 \mathrm{MgCl}_{2}, 7$ dextrose, 1.3 ascorbic acid, and 3 sodium pyruvate), and was decapitated. In the presence of the cutting solution, the brain was removed and near-horizontal hippocampal slices $(350 \mu \mathrm{m})$ were prepared using a Vibratome (Classic 1000; Vibratome). The slices were transferred to a submerged holding chamber containing oxygenated chamber solution (in mm: $125 \mathrm{NaCl}, 2.5 \mathrm{KCl}, 1.25 \mathrm{NaH}_{2} \mathrm{PO}_{4}, 25$ $\mathrm{NaHCO}_{3}, 2 \mathrm{CaCl}_{2}, 2 \mathrm{MgCl}_{2}, 10$ dextrose, 1.3 ascorbic acid, and 3 sodium pyruvate), and were incubated for at least $1 \mathrm{~h}$ before recordings were performed.

Electrophysiology. Neurons were visualized with differential interference contrast microscopy using a Carl Zeiss Axioskop microscope, fitted with a $60 \times$ (Olympus) water-immersion objective. Whole-cell patch recordings in current-clamp mode were made from the soma or the dendrites of CA1 pyramidal neurons using a Dagan IX2-700 amplifier. Signals were low-pass filtered at $5 \mathrm{kHz}$ and sampled at $10-50 \mathrm{kHz}$. Data acquisition and analysis were performed with custom-written software in the Igor Pro environment (Wavemetrics). All recordings were performed at $33-35^{\circ} \mathrm{C}$. The standard extracellular recording solution was made of the following (in mM): $125 \mathrm{NaCl}, 3 \mathrm{KCl}, 1.25 \mathrm{NaH}_{2} \mathrm{PO}_{4}, 25 \mathrm{NaHCO}_{3}, 2$ $\mathrm{CaCl}_{2}, 1 \mathrm{MgCl}_{2}$, and 10 dextrose. Characterization experiments (see Figs. 4-6) were performed with $10 \mu \mathrm{M}$ CNQX, $10 \mu \mathrm{M}$ (+)bicuculline, $10 \mu \mathrm{M}$ picrotoxin, $50 \mu \mathrm{M}$ D,L-APV, and $2 \mu \mathrm{M}$ (2S)-3-[[(1S)-1-(3,4dichlorophenyl)ethyl] amino-2-hydroxypropyl] (phenylmethyl)phosphinic acid (CGP55845) added to the standard solution. Antidromic theta burst pairing (ATBP) (see Figs. 7, 8) experiments were performed with $10 \mu \mathrm{M}$ (+)bicuculline, $10 \mu \mathrm{M}$ picrotoxin, and $2 \mu \mathrm{M}$ CGP55845 in the standard solution. Whenever GABA receptors were blocked, area CA3 was removed to diminish repetitive firing. Electrodes were pulled from borosilicate glass, and their resistance was 4-6 M $\Omega$ for somatic recordings and 5-7 M $\Omega$ for dendritic recordings. The whole-cell recording pipette solution contained the following (in mM): $120 \mathrm{~K}$-gluconate, $20 \mathrm{KCl}, 10 \mathrm{HEPES}, 2 \mathrm{NaCl}, 4$ MgATP, 0.3 Tris-GTP, 7 Tris-phosphocreatine, $\mathrm{pH}$ 7.3. During the course of the experiments, an estimate of the input resistance of the neuron (denoted by $\bar{R}_{\text {in }}$ ) was measured from the steady-state response of the cell to a $100 \mathrm{pA}$ hyperpolarizing current pulse, which was also used to monitor and compensate for changes in series resistance. Access resistance was 10-25 $\mathrm{M} \Omega$ for somatic recordings, and 20-40 M $\Omega$ for dendritic recordings through the period of the experiment. Voltages have not been corrected for the theoretical liquid junction potential, which was measured to be $\sim 8 \mathrm{mV}$.

Impedance profile measurements. The stimulus used for characterizing the impedance profile (see Figs. 4-6) was a Chirp stimulus [we call it the Chirp stimulus and not the impedence amplitude profile (ZAP) stimulus to avoid confusion between the stimulus and the impedance amplitude profile, defined below], which is a sinusoidal current of constant amplitude, with its frequency linearly spanning $0-20 \mathrm{~Hz}$ in $20 \mathrm{~s}$ (Chirp20). The Chirp stimulus used for plasticity experiments (see Figs. 7, 8) had its frequency linearly span $0-15 \mathrm{~Hz}$ in $15 \mathrm{~s}$ (Chirp15). The reason behind using a shorter version of the Chirp stimulus was to obtain two responses to the stimulus per minute, with enough interstimulus interval to compensate for series resistance changes, if necessary. The magnitude of the ratio of the Fourier transform of the voltage response to the Fourier transform of the Chirp stimulus formed the ZAP as follows:

$$
|Z(f)|=\sqrt{\left(\operatorname{Re}(Z(f))^{2}+\left(\operatorname{Im}(Z(f))^{2}\right.\right.},
$$

where $\operatorname{Im}(Z(f))$ and $\operatorname{Re}(Z(f))$ were the imaginary and real parts of the impedance $Z(f)$, respectively. The frequency at which the impedance amplitude reached its maximum was the resonance frequency $\left(f_{\mathrm{R}}\right)$. $|Z|_{\max }$ denotes the maximum value of the ZAP, which by definition equals $\left|Z\left(f_{\mathrm{R}}\right)\right|$. Resonance strength $(Q)$ was measured as the ratio of the maximum impedance amplitude to the impedance amplitude at $0.5 \mathrm{~Hz}$ (Hu et al., 2002). The phase of the ratio of the Fourier transform of the voltage response to the Fourier transform of the Chirp stimulus formed the impedance phase profile (ZPP) as follows:

$$
\phi(f)=\tan ^{-1} \frac{\operatorname{Im}(Z(f))}{\operatorname{Re}(Z(f))} .
$$

Whereas impedance amplitude (see Fig. $1 B$ ) and phase (see Fig. $1 C$ ) as functions of frequency form one way to visualize complex impedances (Middendorf, 1965; Skilling, 1965; Gutfreund et al., 1995), the other way is to use the reactance-resistance locus plot (Cole and Baker, 1941; Middendorf, 1965; Skilling, 1965). See supplemental Figure $1 F$ (available at www.jneurosci.org as supplemental material) for the reactance-resistance locus plot of the RCL circuit shown in Figure $1 \mathrm{~A}$. Given that the focus of this study is on impedance phase, we will use the phase-frequency plot throughout, because it also carries functional relevance in terms of the phase difference between the input current and the output voltage at various frequencies. The total inductive phase was defined as the area under the inductive part of the ZPP (see Fig. $3 B$ ) as follows:

$$
\Phi_{L}=\int_{\phi(f)>0} \phi(f) \mathrm{df} .
$$

Input capacitance estimation. The local input impedance of a neuron encompasses a resistive and a reactive component. In a slice treated with 4-ethylphenylamino-1,2-dimethyl-6-methylaminopyrimidinium chloride (ZD7288), this reactive component is capacitive across all frequencies (see Fig. 5B). To obtain an estimate of this local input capacitance, we computed local input resistance and membrane time constant using two independent techniques, based on recordings from various locations along the somatoapical trunk. The first method, in the frequency domain, involves fitting a Lorentzian of the following form:

$$
\operatorname{Lor}(\omega)=A+\frac{B}{\sqrt{\omega^{2}+\omega_{c}^{2}}}
$$

to the ZAP of a soma/dendrite. The membrane time constant was obtained as the inverse of $\omega_{c}$, the cutoff frequency for the low-pass filter that the ZAP depicts (see Fig. $6 \mathrm{~B}$ ). An estimate of input resistance was computed as the value of the Lorentzian fit at $\omega=0$. The second method, in the time domain (see Fig. $6 \mathrm{C}$ ), involved fitting a double exponential to the charging curve of the somatic/dendritic location responding to a hyperpolarizing step current injection $(100 \mathrm{pA})$. The slower of the two exponential time constants was taken as the membrane time constant (Spruston et al., 1994). An estimate of input resistance, $\bar{R}_{\mathrm{in}}$, was measured from the steady-state voltage response to the current pulse. The local input capacitance $C_{\mathrm{in}}$, for both time and frequency domain techniques, 
A

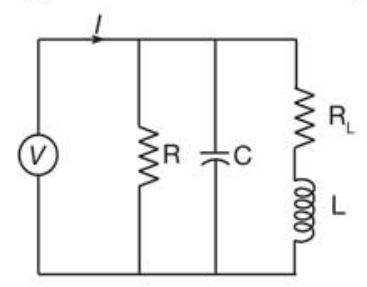

B

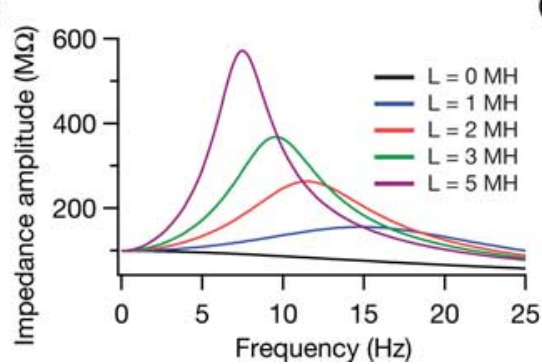

C

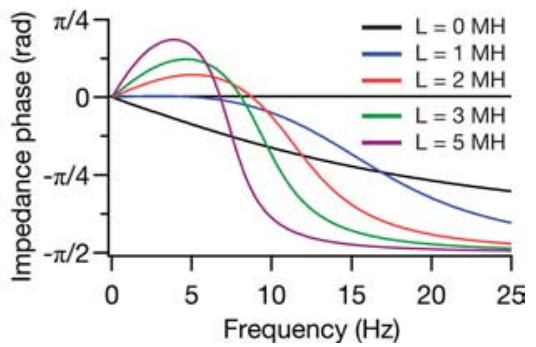

Figure 1. $\quad \boldsymbol{A}, \mathrm{RCL}$ circuit with a leaky inductor. The leak component of the inductor is represented as a resistance in series, $R_{L}$. The values of parameters used for plots in $\boldsymbol{B}$ and $C$ were $R=10 \mathrm{G} \Omega$, $C=90 \mathrm{pF}$, and $R_{L}=100 \mathrm{M} \Omega$. $B$, Impedance amplitude profiles for various values of $L$. For nonzero values of $L$, the circuit acts as a bandpass filter, and its resonance frequency increases with reduction in $L . C$, Impedance phase profile for various values of $L$. For nonzero values of $L$, the impedance phase is positive up to a certain crossover frequency, after which it turns negative. It may be noted that the peak positive phase value decreases with reduction in $L$. See supplemental Figure 1 (available at www.jneurosci.org as supplemental material) for the impedance amplitude and phase profiles of a RCL circuit with an ideal inductor. Plots in $\boldsymbol{B}$ and $\boldsymbol{C}$ were obtained from analytical expressions for impedance amplitude and phase of the circuit in $\boldsymbol{A}$.

was computed as the ratio between the membrane time constant and the input resistance.

In a recent study (Narayanan and Johnston, 2007), we used analysis of the ZAP (Eq. 1) and arrived at location and activity dependence of resonance properties of CA1 pyramidal neurons. In this study, we look at the ZPPs (Eq. 2) and develop a new framework for the analysis and interpretation of intrinsic phase responses of neurons. Although impedance phase and analysis of phase profiles are established techniques (Middendorf, 1965; Skilling, 1965), they have not been applied toward quantitative characterization of neuronal phase responses or phenomenological reactances or dendritic information processing. Frequency-domain impedance analysis mechanisms that we derived and developed from these established techniques and used here provide a broad framework that can be used in understanding intrinsic phase response dynamics and timing control, apart from their parametric dependencies with respect to underlying phenomenological reactances. Furthermore, they also provide a means to understand dendritic information processing, thus providing a quantitative handle on the role that active dendrites play in timing control within neurons. Although substantially new and additional data and analyses (as outlined above) have been included in this study, some of the results presented in this study were derived from the same recordings used in our previous study (Narayanan and Johnston, 2007).

Plasticity protocols. The experimental protocol for the long-term potentiation (LTP) experiments (see Fig. 7) involved an establishment of 5 min stable baselines of evoked EPSPs (3-5 mV peak), $f_{\mathrm{R}}$, and $\bar{R}_{\mathrm{in}}$. EPSPs were evoked by orthodromic stimulation through a bipolar tungsten electrode (O. Stim) (see Fig. 7A) located at $\sim 125 \mu \mathrm{m}$ from the soma, regardless of the location of the recording electrode (see Fig. 7). Action potentials were elicited through a bipolar tungsten electrode (A. Stim) (see Fig. $7 A$ ) placed in the alveus. Induction of plasticity was performed by theta-burst pairing of orthodromic (O. Stim) and antidromic stimulation (A. Stim), with former preceding the latter by $5 \mathrm{~ms}$ (Narayanan and Johnston, 2007). Responses to the Chirp15 stimulus and evoked EPSPs were measured for 40 min after induction of plasticity. Recordings were performed at the initial resting membrane potential throughout the course of the experiment.

Data analysis. In characterization experiments (see Figs. 2, 3), total inductive phase $\left(\Phi_{L}\right)$ values at each membrane voltage were averages of measurements obtained by injecting the Chirp20 stimulus five times. In ATBP experiments (see Figs. 7, 8), baseline values of $\Phi_{L}$ were obtained by averaging measurements over the entire $5 \mathrm{~min}$ baseline period, and postplasticity values were obtained by averaging measurements from the 35-40 min period after induction of plasticity. Group data are expressed as mean \pm SEM. Depending on the dataset, statistical significance was calculated using paired or unpaired Student's $t$ tests, ANOVA, or the Kruskal-Wallis tests. Post hoc analysis following significance with the Kruskal-Wallis test was performed with Dunn's test.

Computer simulations. Simulations were performed using the NEURON simulation environment (Carnevale and Hines, 2006). Integration time step for all simulations was set at $25 \mu \mathrm{s}$. Temperature was set at $34^{\circ} \mathrm{C}$. All simulations were performed on a single compartment model, a cyl- inder of $100 \mu \mathrm{m}$ length and $100 \mu \mathrm{m}$ diameter. Unless otherwise stated, $R_{\mathrm{m}}$ was set as $30 \mathrm{k} \Omega-\mathrm{cm}^{2}, C_{\mathrm{m}}$ was $1 \mu \mathrm{F} / \mathrm{cm}^{2}$, and simulations were performed at $-65 \mathrm{mV}$. Chirp25 current stimulus (see Fig. $2 \mathrm{~B}$, top), a sinusoid spanning $0-25 \mathrm{~Hz}$ in $25 \mathrm{~s}$, was injected to obtain measurements of $\Phi_{L}$ under various parametric variations. The kinetics of the only active mechanism in the model, the $\mathrm{h}$ channel, was set in accordance with experimental measurements from the CA1 pyramidal neuron (Magee, 1998). Specifically, the current through the $\mathrm{h}$ channel, $I_{\mathrm{h}}$, is modeled as follows (see Fig. $2 \mathrm{~A}$ ):

$$
I_{h}=\bar{g}_{\mathrm{h}} s\left(V_{m}, t\right)\left(V_{m}-E_{\mathrm{h}}\right),
$$

where $\bar{g}_{\mathrm{h}}$ denotes maximal h conductance; $V_{\mathrm{m}}$, the membrane voltage; and $E_{\mathrm{h}}$, the reversal potential for the h channel. $s\left(V_{\mathrm{m}}, t\right)$ controls the voltage dependence and kinetics of the $h$ channel, and evolves based on first-order kinetics (Gasparini et al., 2004) as follows:

$$
\begin{gathered}
\frac{d s}{d t}=\frac{s_{\infty}-s}{\tau_{\mathrm{h}}} \\
s_{\infty}(V)=\left(1+\exp \left(\left(V-V_{1 / 2}\right) / 8\right)\right)^{-1} \\
\tau_{\mathrm{h}}(V)=\frac{\exp (0.033(V+75))}{0.011(1+\exp (0.083(V+75))} .
\end{gathered}
$$

The default value of $V_{1 / 2}$ was set at $-82 \mathrm{mV}$ (Magee, 1998).

\section{Results}

Electrical equivalent circuits have been widely used in the representation and analysis of neuronal membrane (Cole, 1932, 1941; Hodgkin and Huxley, 1952). Impedance, a standard measurement for characterizing properties of neuronal membrane represented through such circuits, is a complex quantity comprised of resistance as its real part and reactance as its imaginary part. Whereas negative reactances are offered by capacitive elements, inductive elements contribute to positive reactances. As a complex number, impedance can also be represented using the magnitude-phase form, with these two forms of representations related to each other as in Equations 1-2. Analysis of the impedance amplitude offered by a circuit constructed with all three basic components (Fig. 1A), resistance, capacitance, and inductance, indicates that such a circuit can act as a bandpass filter (Fig. $1 B$ ). The bandpass property of such a circuit owes its frequency dependence to the combined presence of the high-pass RL circuit and the low-pass RC circuit (Middendorf, 1965; Skilling, 1965). Analysis of the phase component of the impedance offered by such a circuit, however, illustrates the temporal relationship between the injected current and the steady-state voltage response (Fig. 1C). Specifically, for nonzero values of the inductance $L$, the steady-state voltage response leads the current up to a crossover 


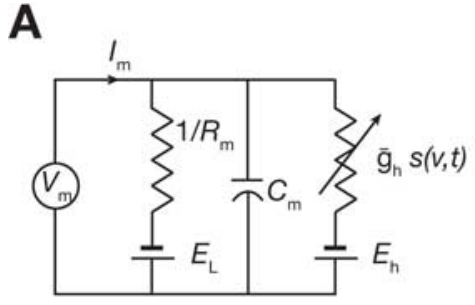

\section{C}

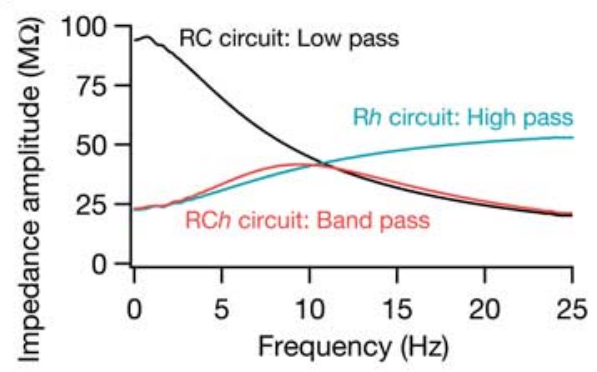

E

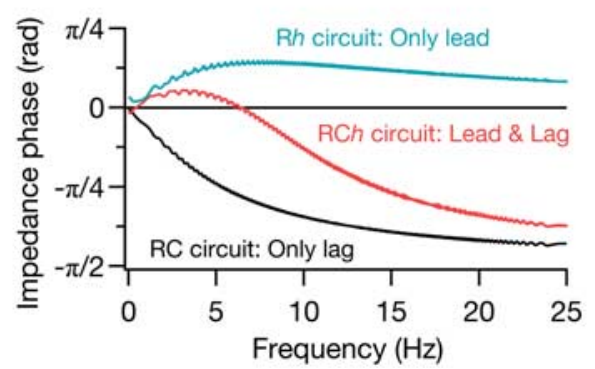

B

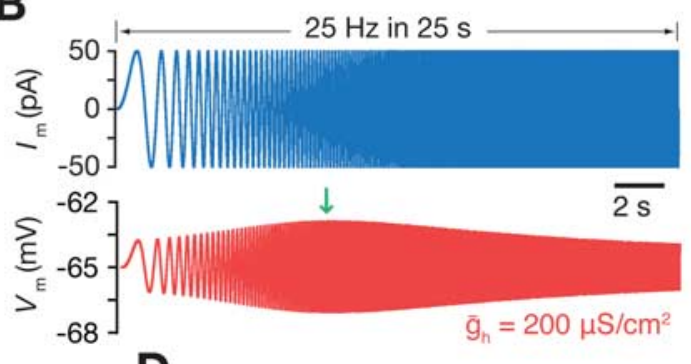

D

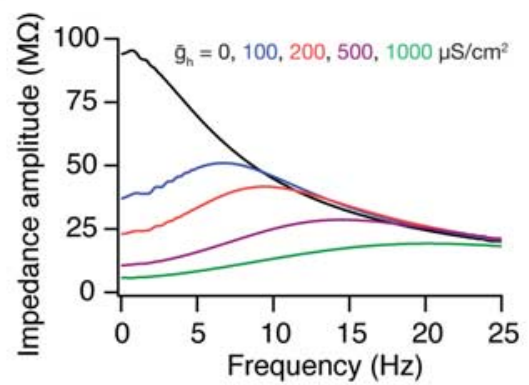

F

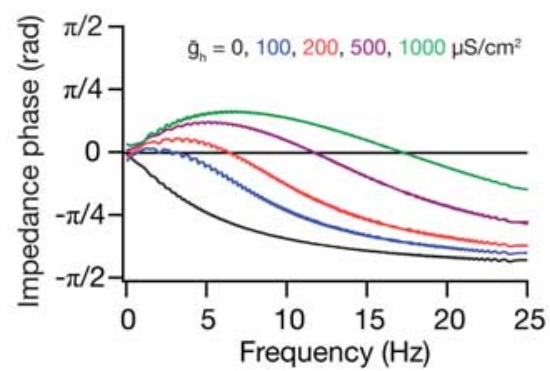

G

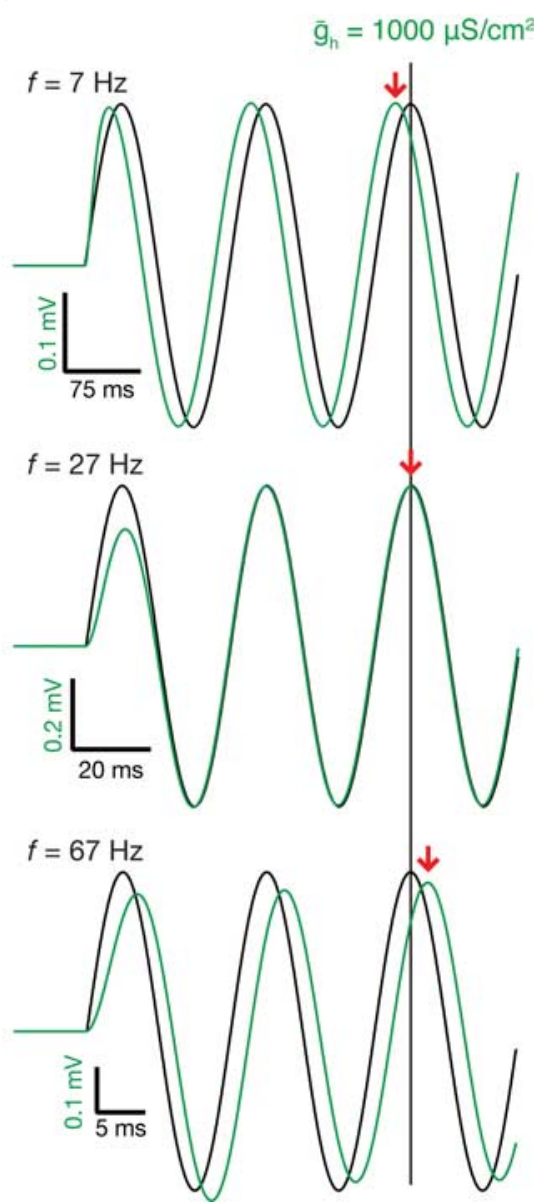

Figure 2. Simulations suggest that the $h$ channel contributes an inductive component to neuronal input impedance. $A$, RCh circuit representing a single compartment neuron model, with the $h$ conductance as the only active mechanism. $R_{\mathrm{m}}$ and $C_{\mathrm{m}}$ represent specific membrane resisitivity and specific membrane capacitance, respectively; $\bar{g}_{\mathrm{h}}$ is the maximal $\mathrm{h}$ conductance and $s(V, t)$ is the state variable that models the voltage dependence and kinetics of the $\mathrm{h}$ conductance. $\boldsymbol{B}$, Chirp25 current stimulus (top) and a typical voltage response (bottom) of the model in $\boldsymbol{A}$. $\boldsymbol{C}$, Impedance amplitude profiles of the $R C$ circuit (with $\bar{g}_{\mathrm{h}}=0$ in $\boldsymbol{A}$ ), the Rh circuit (with $C_{\mathrm{m}}=0$ in $\boldsymbol{A}$ ), and the RCh circuit display low-pass, high-pass, and bandpass characteristics, respectively. For Rh and RCh plots, $\bar{g}_{\mathrm{h}}=200 \mu \mathrm{S} / \mathrm{cm}^{2}$.D. Impedance amplitude profiles of the RCh circuit obtained with various values of $\bar{g}_{\mathrm{h}}$. Note that the peak impedance amplitude decreases and the frequency at which this peak occurs (the resonance frequency) increases with increase in $\bar{g}_{\mathrm{h}}$. $\boldsymbol{E}$, Impedance phase profiles of the Rh circuit, the RC circuit, and the RCh circuit display positive, negative, and positive-negative impedance phases, respectively. For Rh and RCh plots, $\bar{g}_{\mathrm{h}}=200 \mu \mathrm{S} / \mathrm{cm}^{2} . \boldsymbol{F}$, Impedance phase profiles of the RCh circuit obtained with various values of $\bar{g}_{\mathrm{h}}$. Note that both the peak positive phase value and the frequency at which the crossover from positive to negative phase occurs increase with increase in $\bar{g}_{\mathrm{h}}$. $\mathbf{G}$, Voltage responses (green) of the RCh circuit to sinusoidal currents (black; 100 pA peak to peak) of various frequencies illustrate the lead in the steady-state voltage response at lower frequencies followed by the lag at higher frequencies. It may be noted that the steady-state response evolves over the first few cycles because of the interplay between activation/deactivation of h channels and the passive $R C$ circuit. The red arrows indicate the locations of the voltage peak for the third cycle; their locations may be compared with the peak of the current injection (the black line) to infer the lead-lag behavior spanning input frequencies. Simulations in $\mathbf{G}$ were performed at $-75 \mathrm{mV}$, whereas the other simulations were performed at the default $-65 \mathrm{mV}$.

frequency after which there is a lag in the voltage response with respect to the current injected (Fig. 1C). This phenomenon is attributable to the conjunctive action of the RL circuit, which offers positive phase values (voltage leads current) at all frequencies and the RC circuit, which always contributes negative phase values (voltage lags current) at all frequencies (Middendorf, 1965; Skilling, 1965). Positive impedance phase and positive reactance (Eq. 2) are considered as traditional hallmarks for the presence of an inductor in the circuit (Middendorf, 1965; Skilling, 1965).

In a recent study (Narayanan and Johnston, 2007) analyzing impedance amplitude, we had established that the resonance properties of a CA1 pyramidal neuron are location dependent and plastic. In this study, we employ impedance phase to analyze the presence, basis, dependencies, and plasticity of the inductive component of the local input impedance [for analyses of transfer impedance, which represents filtering along the dendritic cable, as opposed to input impedance, which characterizes local neuronal response to external inputs, see Ulrich (2002) and Rumsey et al. (2007)]. We accomplish this by measuring voltage responses elicited by sinusoidal current injection (Chirp stimulus) (see Materials and Methods) to various locations of these neurons.

\section{The $h$ channel contributes an inductive reactance to neuronal input impedance}

To understand the $\mathrm{h}$ conductance in terms of the impedance it offers, we constructed a single compartment model of neuronal membrane and included the $\mathrm{h}$ conductance as the only active mechanism to the model (Fig. $2 A$ ). We computed the input impedance of this model by measuring its voltage response to the Chirp25 current signal (Fig. 2 B). Our simulations indicated that, whereas the RC circuit acted as a low-pass filter, the Rh circuit behaved as a high-pass filter (Fig. 2C), indicating that the h conductance is behaving similar to an inductive element (compare 
Fig. $1 B$ ). Consistent with this, the RCh circuit acted as a bandpass filter (Fig. 2C), with $f_{\mathrm{R}}$ increasing with increasing values of the $\mathrm{h}$ conductance (Fig. 2D) (Hutcheon and Yarom, 2000; Narayanan and Johnston, 2007). We then analyzed the phase component of the input impedance to check whether the $\mathrm{h}$ conductance also contributed to the voltage response leading the current input. We observed that, at all measured frequencies, the Rh circuit provided a positive phase response in contrast to the $\mathrm{RC}$ circuit, which provided a negative phase (Fig. $2 E$ ). When all the three components of the circuit were present, we observed positive phases at frequencies lower than a crossover frequency, above which the impedance phase was negative (Fig. 2 F; compare Fig. $1 C)$. The presence of positive reactance component in the input impedance may also be noted from the reactance-frequency plot (supplemental Fig. $1 \mathrm{H}$, available at www.jneurosci.org as supplemental material) and reactance-resistance plot (supplemental Fig. 1 , available at www.jneurosci.org as supplemental material).

We also confirmed that the lead-lag phase response could be obtained with injection of individual sinusoids of various frequencies, rather than using the Chirp stimulus (Fig. 2G). The first few response cycles to sinusoids at various frequencies also provide an intuitive understanding of the phase lead observed at lower frequencies, established just from the slow kinetics and the voltage dependence of the $\mathrm{h}$ channel (Magee, 1998). At lower frequencies, in response to the depolarizing phase of a sine wave current injection, the cell depolarizes and induces deactivation of the $\mathrm{h}$ channel. This turns off the inward h current, thus causing the voltage peak to occur before the peak of the sine wave current. As the sine wave begins to repolarize, the inward h current turns on, causing the voltage trough to occur before the current trough. Over a few cycles, because of the interplay between the h-channel kinetics and the passive RC circuit, the steady-state lead in phase is fixed to a certain angle, and the voltage response continues to lead the injected current by that angle. This deactivation/activation cycle also allows the $\mathrm{h}$ current to reduce the voltage response at these lower frequencies, thus reducing the impedance amplitude as well (Fig. 2G, $7 \mathrm{~Hz}$ ). At higher frequencies, however, because of the slow kinetics of the h channel, there is not enough time for the channel to activate or deactivate during a single half-cycle. This allows the passive capacitive component to dominate, with the voltage charging up to a maximum value, and thus lagging the injected current. Because the h current activation/ deactivation is less at higher frequencies, its contribution to the peak voltage is also lesser, making the membrane completely dependent on the RC circuit (Fig. $2 G, 67 \mathrm{~Hz}$ ). At the crossover frequency (Fig. 2G, $27 \mathrm{~Hz}$ ), the lead offered by the h current and the lag contributed by the capacitive component cancel each other, letting the current and the voltage response to be in phase with each other. Together, these simulations suggest that the $h$ conductance contributes an inductive component to the input impedance of the neuron it resides in. Because the functional expression of this inductive component is dependent on the voltage dependence and kinetics of the h conductance and not attributable to the presence of a real inductor in the neuron, the $h$ channel functionally expresses a phenomenological inductance (Mauro et al., 1970; Koch, 1984).

Although the overall responses are similar, the differences between the RCL circuit responses (Fig. 1) and the RCh circuit responses (Fig. 2) could be attributed to a number of factors: (1) decrease in $L$ (Fig. $1 B$ ) and increase in $\bar{g}_{\mathrm{h}}$ (Fig. $2 D$ ) lead to an increase in $f_{\mathrm{R}}$, because $L$ is directly proportional to the reactance, whereas $\bar{g}_{\mathrm{h}}$ is a conductance measure, which is inverse of resistance; (2) the difference in impedance amplitude at $f=0$ is ob- served only with change in $\bar{g}_{\mathrm{h}}$ of the RCh circuit, and not with change in $L$ of the RCL circuit. This is because any phenomenological inductance, and thus the h conductance, contributes not just to the $L$ component (compare supplemental Fig. 1, available at www.jneurosci.org as supplemental material), but to both $R_{L}$ and $L$, and to the chord conductance of the channel that is in parallel to these two components (Mauro et al., 1970; Puil et al., 1988). The differences in the phase plots of the RCL and RCh also could be attributed to these very reasons. Taking these together, the $h$ conductance could be considered as equivalent to an inverse leaky inductance, which also contributes to steady-state input resistance. For analyses of similar models, see Puil et al. (1988) and Hutcheon et al. (1996b).

\section{Inductive phase is dependent on passive and h-channel properties}

A glance at the phase plots (Fig. $2 F$ ) suggests that the crossover frequency (from positive to negative phase) and the peak positive phase of the input impedance increased with increase in $\bar{g}_{\mathrm{h}}$. To quantify the inductive component of input impedance across various parameters of the RCh circuit (Fig. 3A), we devised a measure that would be sensitive to both the extent and the amount of positive phase in a given impedance profile. We called this measure the total inductive phase, $\Phi_{L}$, and defined it as the area under the positive segment of the impedance phase profile (Fig. 3B, Eq. 3). Because positive impedance phases are contributed by positive reactances (Eq. 2), this measure would also be a measure of the total inductive reactance in an impedance profile.

Using simulations on the RCh circuit, we found that $\Phi_{L}$ increased monotonically with $\bar{g}_{\mathrm{h}}$ (Fig. $3 C$ ), whereas it had a bellshaped dependence on both the membrane voltage (Fig. 3D) and the half-maximal activation voltage for the $\mathrm{h}$ conductance (Fig. $3 E)$.

Phenomenological inductances (alternately called resonating conductances) are contributed by conductances that actively oppose changes in membrane voltage (Cole, 1968; Sabah and Leibovic, 1969; Hutcheon and Yarom, 2000). The functional expression of a phenomenological inductance is not only dependent on the kinetics and voltage dependence of the mediating ion channel, but also on the passive properties of the membrane they reside in (Table 1). Specifically, for this inductance to functionally express itself, the passive time constant of the neuron should be less than the activation/deactivation time constant of the ion channel mediating it (Hutcheon et al., 1996b; Hutcheon and Yarom, 2000). Consistent with this, $\Phi_{L}$ (Fig. $3 F$ ), $f_{\mathrm{R}}$ (supplemental Fig. 3D, available at www.jneurosci.org as supplemental material), and $Q$ (supplemental Fig. $3 E$, available at www.jneurosci.org as supplemental material) exhibit a threshold on the activation/deactivation time constant, below which the membrane acts noninductive ( $\Phi_{L}$ is zero and resonance is abolished). Beyond that threshold, there was a steep rise and slow decay in $\Phi_{L}$ with increase in $\tau_{\mathrm{h}}$ (Fig. $3 F$ ). Regarding passive properties, $\Phi_{L}$ increased with specific membrane resistivity (Fig. $3 G$ ), and decreased sharply with increase in specific membrane capacitance (Fig. $3 H$ ). Thus, although positive phase responses and bandpass amplitude responses (Fig. 2) are indicative of the presence of an ion channel that can behave as a phenomenological inductance, the absence of these measures is not proof for the absence of that ion channel in a given neuron. Even in the presence of a given ion channel, passive properties of the neuron and the time constants of the channel in that neuron play crucial roles in its functional expression as an inductance (Hutcheon and Yarom, 2000).

From these analyses, it is clear that the amplitude and phase of 
A

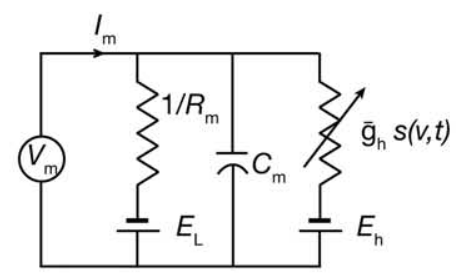

B

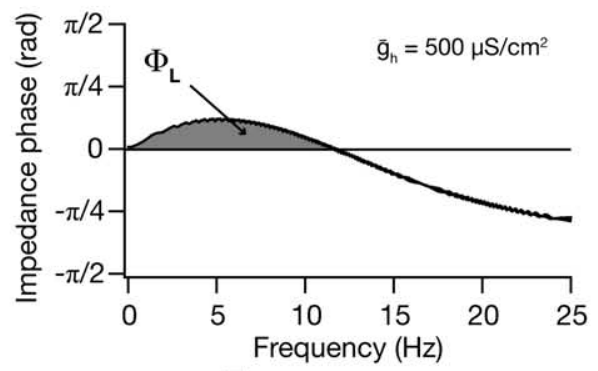

C

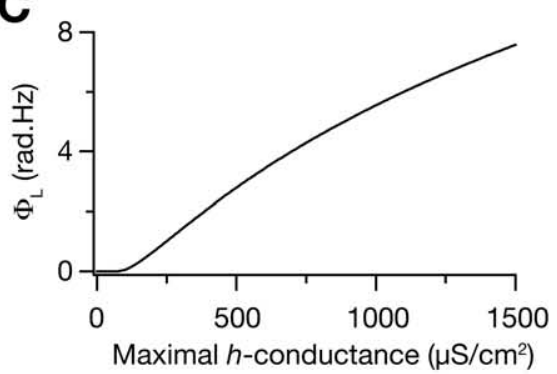

$\mathbf{F}$

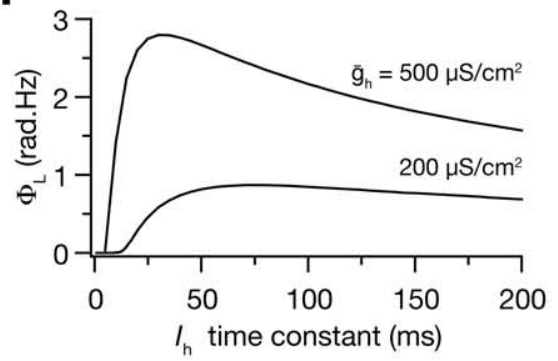

D

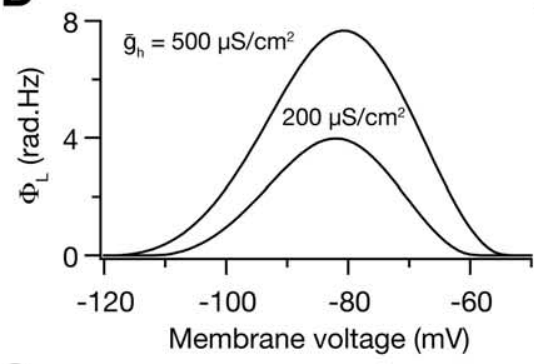

G

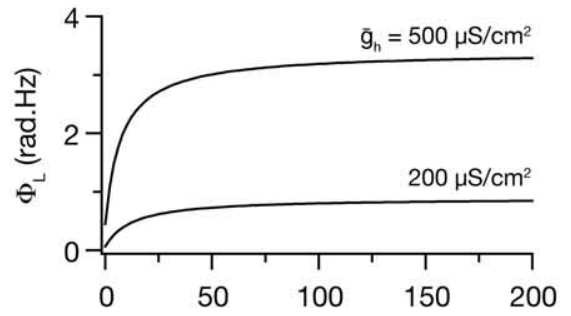

Specific membrane resistivity $\left(\mathrm{k} \Omega-\mathrm{cm}^{2}\right)$
E

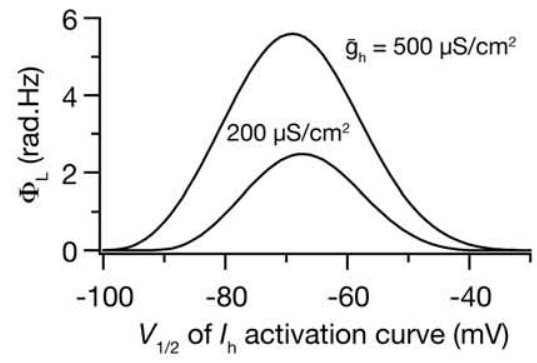

H

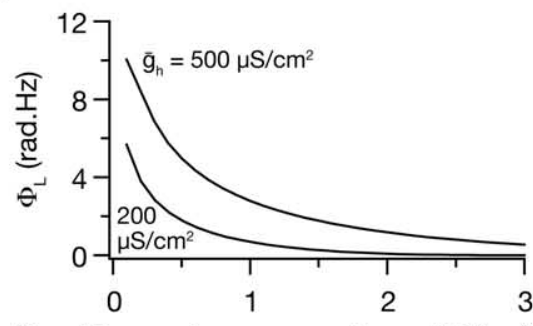

Figure 3. Simulations suggest that the inductive phase component of the $R C h$ circuit is dependent on passive and h-channel properties. $A$, RCh circuit representing a single compartment neuron model, with the h conductance as the only active mechanism. $\boldsymbol{B}$, lllustration of the $\Phi_{L}$ measurement. The plot shows the impedance phase profile of the RCh obtained with maximal $\mathrm{h}$ conductance, $\bar{g}_{h}$, set at $500 \mu \mathrm{S} / \mathrm{cm}^{2}$ (also in Fig. 2F). The shaded region represents the $\Phi_{L}$ and was computed as in Equation 3. $\Phi_{L}$ as functions of $\bar{g}_{\mathrm{h}}(\boldsymbol{C})$, membrane voltage (D), voltage at which half-maximal activation occurs for the $h$ channel $(\boldsymbol{E})$, activation/deactivation time constant of the $h$ channel $(\boldsymbol{F})$, specific membrane resistivity $(\boldsymbol{G})$, and capacitance $(\boldsymbol{H})$. $\boldsymbol{D}-\boldsymbol{H}$ are plotted for two different values of $\bar{g}_{\mathrm{h}}$ to illustrate that each of these plots scale up with increase in $\bar{g}_{\mathrm{h}}(\boldsymbol{C})$, without change in their respective functional forms. The dependencies, with respect to each of these parameters, of impedance amplitude and phase profiles and various measurements associated with them are provided in supplemental Figures $2-4$ (available at www.jneurosci.org as supplemental material) and Table 1.

Table 1. Effects on steady-state input resistance $\left(R_{\text {in }}\right)$, maximum impedance amplitude $\left(|Z|_{\max }\right)$, resonance frequency $\left(f_{\mathrm{R}}\right)$, resonance strength $(Q)$, and total inductive phase $\left(\Phi_{L}\right)$ with increases in various parameters associated with the RCh circuit (Fig. 2A)

\begin{tabular}{|c|c|c|c|c|c|}
\hline \multirow[b]{2}{*}{ Increase in } & \multicolumn{5}{|l|}{ Effects on } \\
\hline & $R_{\text {in }}$ & $|z|_{\max }$ & $f_{\mathrm{R}}$ & 0 & $\Phi_{L}$ \\
\hline$V_{m}$ & Increase & Increase & Bell-shaped & Bell-shaped & Bell-shaped \\
\hline$R_{\mathrm{m}}^{\mathrm{m}}$ & Increase & Increase & Decrease & Increase & Increase \\
\hline$C_{m}$ & No change & Decrease & Decrease & Decrease & Decrease \\
\hline $\bar{g}_{h}$ & Decrease & Decrease & Increase & Increase & Increase \\
\hline$V_{1 / 2}$ & Decrease & Decrease & Bell-shaped & Bell-shaped & Bell-shaped \\
\hline$\tau_{\mathrm{h}}$ & No change & Increase & Decrease $^{a}$ & Nonmonotonic & Nonmonotonic \\
\hline
\end{tabular}

${ }^{a}$ There exists a minimal activation/deactivation time constant, $\tau_{\mathrm{h}}$, required for a neuron to resonate (Hutcheon et al., 1996b; Hutcheon and Yarom, 2000), but after $\tau_{\mathrm{h}}$ crossed that threshold and the neuron does resonate, the resonance frequency decreases with increase in $\tau_{\mathrm{h}}$. This threshold value on $\tau_{\mathrm{h}}$ also reduces with increase in $\bar{g}_{h}$ (Fig. 3F; supplemental Fig. 3D,E, available at www.jneurosci.org as supplemental material), elucidating the complex relationships that exist between the passive and h-channel properties. For plots associated with this table, see Figure 3 and supplemental Figures $2-4$ (available at www. jneurosci.org as supplemental material).

input impedance provide quantitative measures of intrinsic excitability and intrinsic phase response of neurons, because they characterize the intrinsic response dynamics of the neuron to input signals at various frequencies. These analyses (Fig. 3; supplemental Figs. 2-4, available at www.jneurosci.org as supplemental material; Table 1) also provide a general framework for analyzing the roles of various physiologically relevant parameters of phenomenological inductances [including their voltage dependence, gradients in density, different subunits of channels exhibiting different kinetics (e.g., HCN1-HCN4 for h channels), their differential modulation and activitydependent plasticity] in regulating intrinsic excitability and phase response properties. In what follows, we experimentally measure the intrinsic phase response of CA1 pyramidal cells, and demonstrate its dependence on voltage, dendritic location, and activity.

\section{Proximal and distal dendrites display distinct input impedance phases}

We first tested the voltage dependence of impedance phase by measuring the responses of the somata and dendrites of CA1 pyramidal neurons to the Chirp20 stimulus (Fig. 4A). Applying the phase analysis we developed in the preceding sections to these experimental responses, we observed an increase in $\Phi_{L}$ with hyperpolarization, in the measured subthreshold voltage range of -75 to $-55 \mathrm{mV}$, across all recording 
A

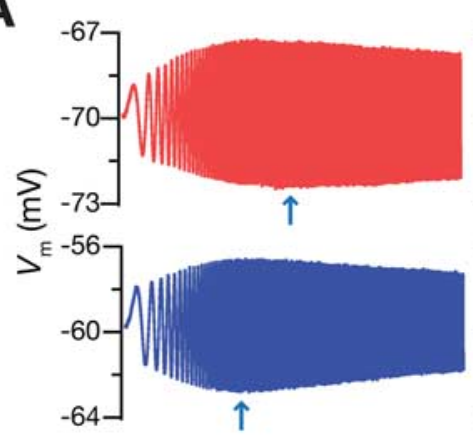

C

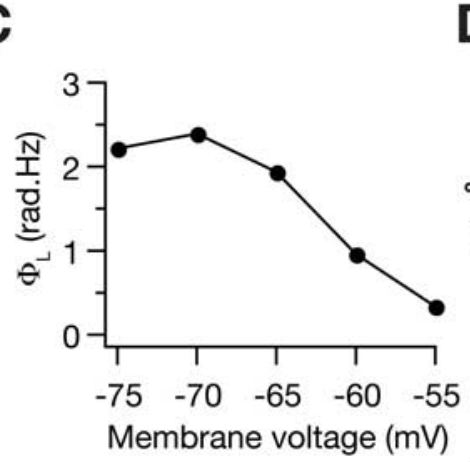

F

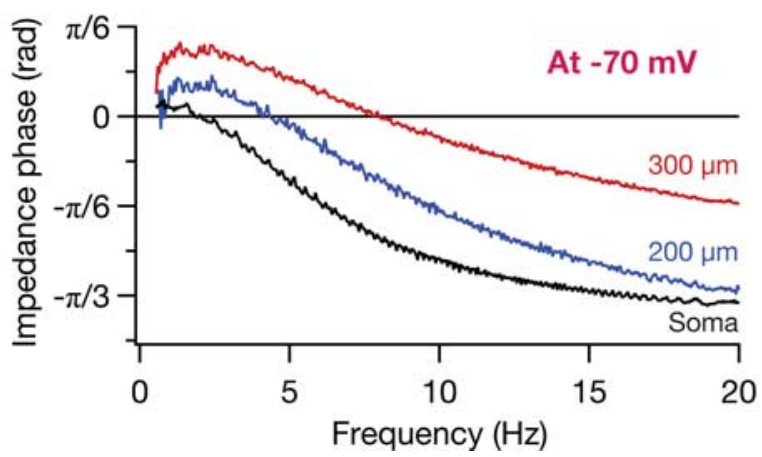

B

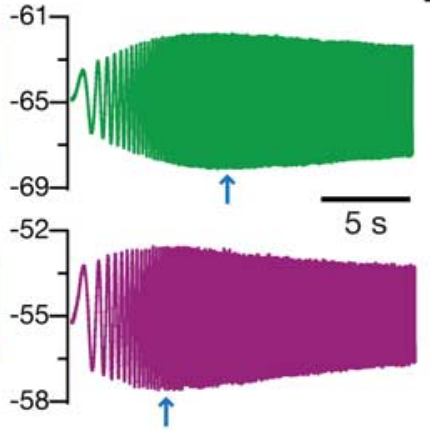

D

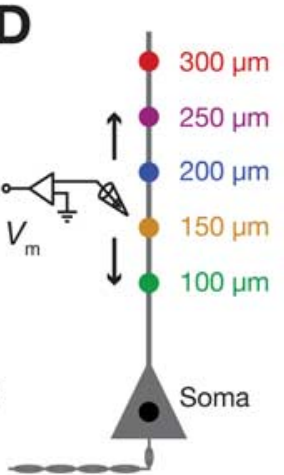

G

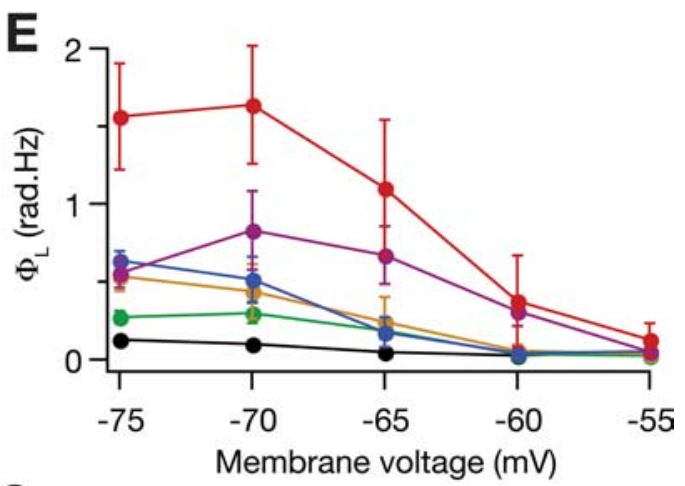

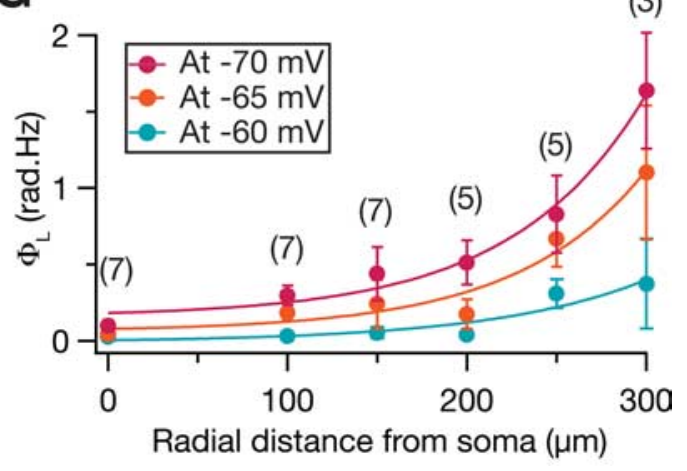

Figure 4. Whole-cell recordings establish the exponential increase in the inductive component of the input impedance with increase in dendritic recording distance from the soma. $\boldsymbol{A}$, Responses of a CA1 pyramidal cell dendrite $320 \mu \mathrm{m}$ away from the soma to the Chirp20 current injection. Each panel depicts the response of the dendrite measured at different membrane voltages, which were set by injecting appropriate depolarizing or hyperpolarizing holding current. The arrows indicate the location of maximal response in each trace. The color code serves as a means to interpret corresponding plots in $\boldsymbol{B}$. $\boldsymbol{B}$, ZPPs obtained from traces shown in subpanels of $\boldsymbol{A}$. Note that both the frequency extent and peak amplitude of positive phase values increase with membrane hyperpolarization. $\boldsymbol{C}$, Total inductive phase of the input impedance $\left(\Phi_{L}\right)$ computed from ZPPs shown in $\boldsymbol{B}$ illustrates its increase with hyperpolarization, quantitatively confirming observations arrived through visual inspection of $\boldsymbol{B}$. Corresponding impedance amplitude profiles and other resonance properties for traces in $\boldsymbol{A}$ are given in supplemental Figure $5, B$ and $C$ (available at www.jneurosci.org as supplemental material). $D$, Schematic of the somatoapical trunk, depicting the experimental design for assessing inductive phase as a function of distance from the soma. Voltage responses of the soma and dendrites at various distances (up to $320 \mu \mathrm{m}$ away from the soma) to the Chirp20 stimulus were recorded locally using a whole-cell patch-clamp electrode ( $V_{\mathrm{m}}$ ). Colors of markers along the somatoapical trunk serve as codes for corresponding distances in $\boldsymbol{E}$ and $\boldsymbol{F}$. $\boldsymbol{E}$, In the measured voltage range of $-75 \mathrm{mV}$ to $-55 \mathrm{mV}$, regardless of distance from soma, total inductive phase of input impedance increases with hyperpolarization. $\boldsymbol{F}$, Typical ZPPs, measured at $-70 \mathrm{mV}$, corresponding to somatic and various dendritic distances, each obtained from different neurons, color-coded as in $\mathbf{D}$. Note that both the frequency extent and peak amplitude of positive phase values increase with distance from soma. $\mathbf{G}$, Total inductive phase of dendritic input impedance increases exponentially (exponential fit: $-70 \mathrm{mV}, \tau=73.53 \mu \mathrm{m} ;-65 \mathrm{mV}, \tau=71.43 \mu \mathrm{m} ;-60 \mathrm{mV}, \tau=86.42 \mu \mathrm{m}$ ) with distance from the soma. The number within parentheses at each distance value is the number of somatic or dendritic recordings performed to arrive at the plots shown in $\boldsymbol{E}$ and $\boldsymbol{G}$. All error bars represent $\mathrm{SEMs}$.

locations along the somatoapical axis (Fig. $4 E$ ). Because $\Phi_{L}$ increases with $\bar{g}_{\mathrm{h}}$ (Fig. $3 C$ ) and h channel density increases with distance from the soma of CA1 pyramidal cells (Magee, 1998; Lorincz et al., 2002), we next tested the hypothesis that $\Phi_{L}$ should increase with distance from the soma. Our results demonstrated that the inductive component of dendritic input impedance increases exponentially with distance from the soma (Fig. $4 F, G$ ). Thus, across the somatoapical trunk, the local voltage response leads the injected current over a range of frequencies, which increased with either hyperpolarization or with increasing distance of recording location from the soma. It should be noted that for inputs in the theta frequency range $(3-10 \mathrm{~Hz})$, even a small phase difference of $\sim \pi / 6$ radians translates to $\sim 8-28 \mathrm{~ms}$, a time difference that is sufficient to impact crucial neuronal functions such as temporal integration, coincidence detection, spike-timing dependent plasticity, and spike initiation.

At more depolarized voltages, another form of resonance, mediated by the M-type potassium current, has been reported in CA1 pyramidal neurons (Hu et al., 2002). In our previous study (Narayanan and Johnston, 2007), we had confirmed the presence 
A

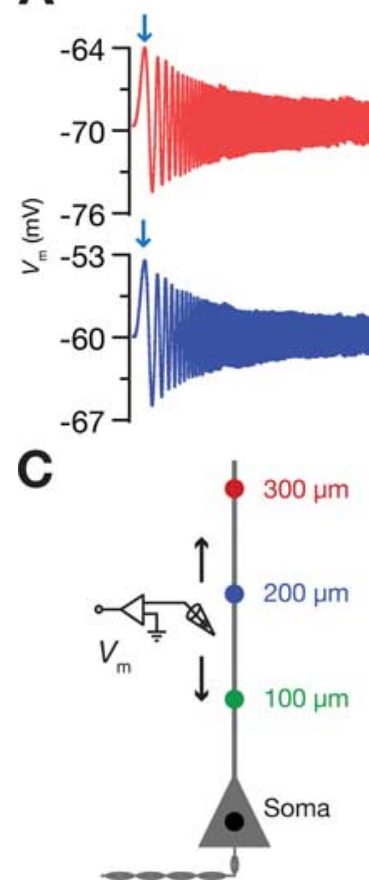

B

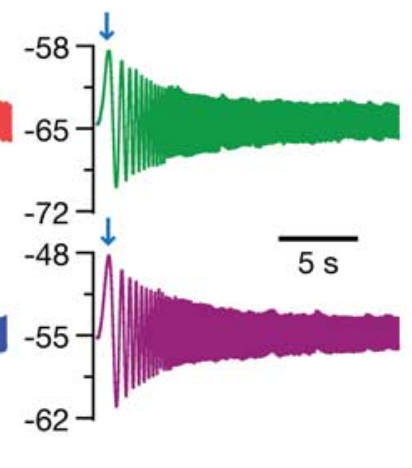

D

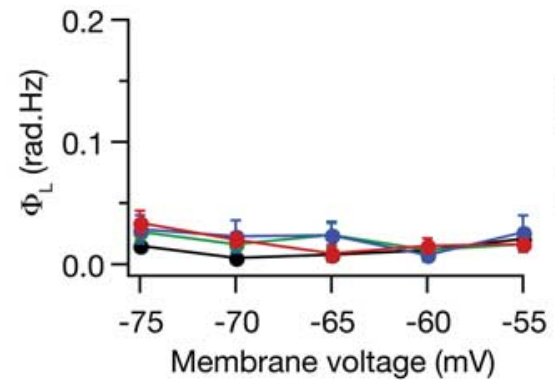

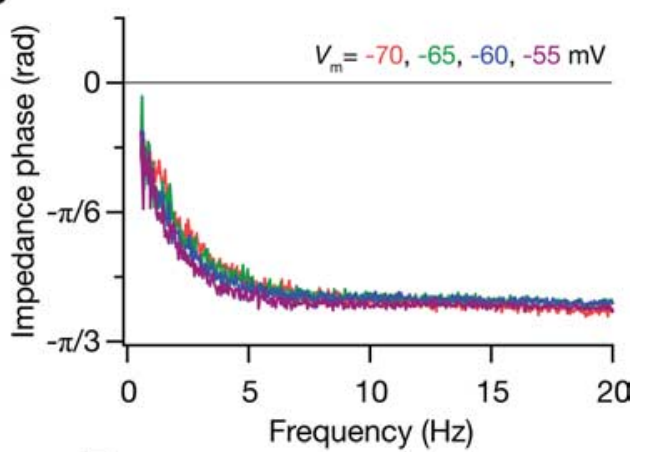

E

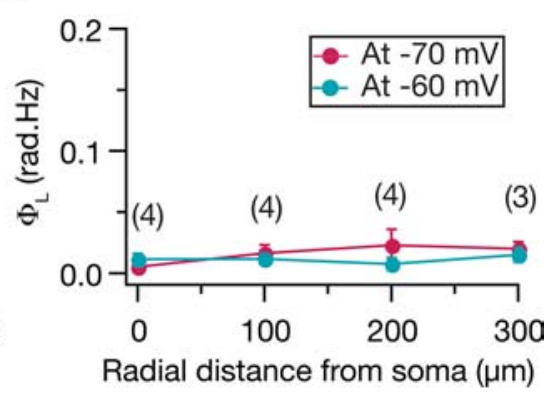

Figure 5. Pretreatment with ZD7288 abolishes the inductive component of input impedance along the somatoapical trunk. A, Responses of a CA1 pyramidal cell dendrite $280 \mu \mathrm{m}$ away from the soma to the Chirp20 current injection. Each panel depicts the response of the dendrite measured at different membrane voltages. The arrows indicate the location of maximal response in each trace. The color code serves as a means to interpret corresponding plots in $\boldsymbol{B} . \boldsymbol{B}$, ZPPs obtained from traces shown in subpanels of $\boldsymbol{A}$. Note that the inductive component is zero for all measured voltages, because the impedance phase never gets positive. Corresponding impedance amplitude profiles for traces in $A$ are given in supplemental Figure $5 D$ (available at www.jneurosci.org as supplemental material). C, Schematic of the somatoapical trunk, with colors of markers serving as codes for corresponding distances in $\boldsymbol{D}$. At all measured voltages (D) and at all distances from the soma (E), the total inductive phase $\left(\Phi_{l}\right)$ of the local input impedance is close to zero. The number within parentheses at each distance value is the number of somatic or dendritic recordings performed to arrive at the plots shown in $\boldsymbol{D}$ and $\boldsymbol{E}$. All error bars represent SEMs.

of this form of resonance using carbachol to block the $\mathrm{M}$ current. Given that all resonating conductances can be considered to be phenomenological inductances, we asked whether the $\mathrm{M}$ conductance also provides a lead in the impedance phase. Our results (supplemental Fig. 5A, available at www.jneurosci.org as supplemental material) suggest that the $M$ conductance indeed contributes an inductive component to the input impedance of CA1 pyramidal neurons. This was confirmed through the carbachol-induced reduction in the inductive phase component specifically at depolarized voltages, but not at more hyperpolarized voltages (supplemental Fig. 5A, available at www.jneurosci.org as supplemental material). In CA1 pyramidal neurons, in striking contrast to the $h$ channel, the $\mathrm{M}$ channel is known to express at higher densities in the perisomatic region than at the distal dendrites (Hu et al., 2007), and the two forms of resonance mediated by these channels exhibit almost nonoverlapping voltage dependencies (Hu et al., 2002). Given these dependencies, our results suggest that the $\mathrm{M}$ channel, like the $\mathrm{h}$ channel, contributes to the voltage and location dependencies of the intrinsic phase response of the neuron. The almost nonoverlapping voltage dependencies and almost inverse gradient of the $\mathrm{M}$ channel compared with the $h$ channel provide an interesting contrast in these two phenomenological inductances that CA1 pyramidal neurons express. Although these results bring forth the generalizability of our analysis techniques, because the focus of our study is on the h channel, we continue to analyze its role in regulating intrinsic phase responses of these neurons.

\section{Distance-dependent gradient in inductive reactance is attributable to changes in $\mathrm{h}$-channel properties}

To test whether the dependencies of $\Phi_{L}$ on voltage and distance were indeed attributable to change in h-channel properties, we pretreated slices for $5 \mathrm{~min}$ with $100 \mu \mathrm{M}$ ZD7288, an irreversible specific blocker of the $\mathrm{h}$ channel (Gasparini and DiFrancesco, 1997 ) and assessed voltage and distance dependencies of $\Phi_{L}$ without adding ZD7288 to the bath. We found that the impedance phase profile reflected that of an RC circuit (compare Fig. 2E), with no positive phase element across all frequencies, thus setting $\Phi_{L}$ to zero at all measured voltages (Fig. $5 A, B$ ). This abolishment of the inductive component in ZD7288-treated slices was confirmed across a range of distances (up to $300 \mu \mathrm{m}$ from the soma) and a range of subthreshold voltages (Fig. $5 D, E$ ). Thus, in the subthreshold voltage range, the h channel exclusively accounts for the inductive component of the input impedance of CA1 pyramidal cell soma and dendrites.

Although pharmacology suggests that the inductive component was mediated by the $h$ conductance, it does not test whether the distance-dependent increase in the inductive component (Fig. $4 F, G$ ) was attributable to changes in passive properties (supplemental Fig. 4, available at www.jneurosci.org as supplemental material; Table 1) along the dendritic cable. For instance, it has been argued in dendrites of cortical pyramidal neurons that the local input capacitance decreases with distance from the soma (Williams and Stuart, 2003). To test whether a similar reduction in local input capacitance could account for the increase in $f_{\mathrm{R}}$ (Narayanan and Johnston, 2007) and in the inductive reactance 


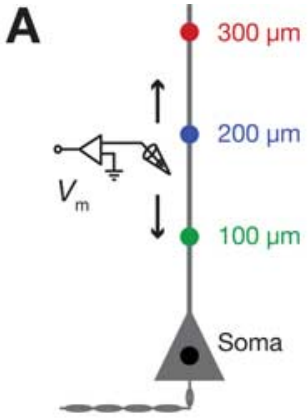

D

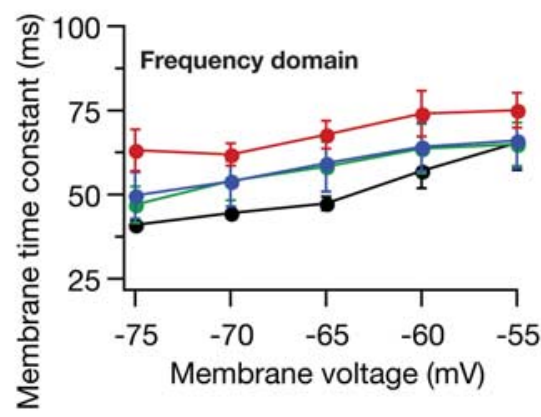

G

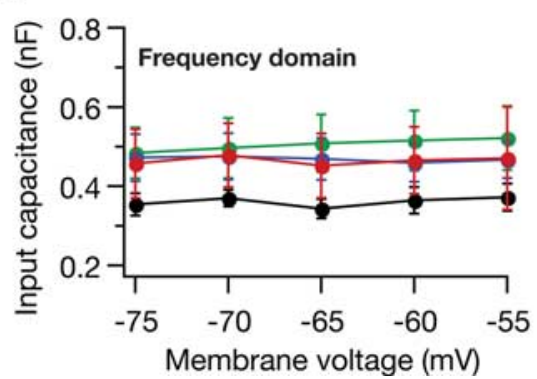

B

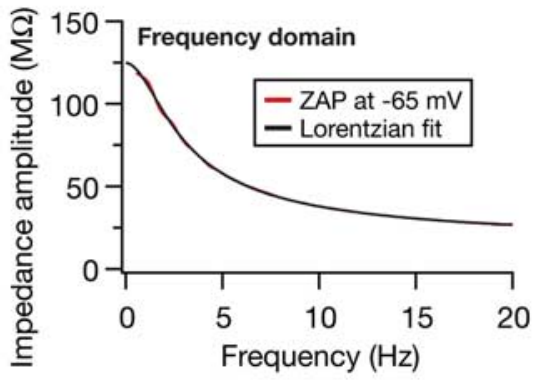

E
C

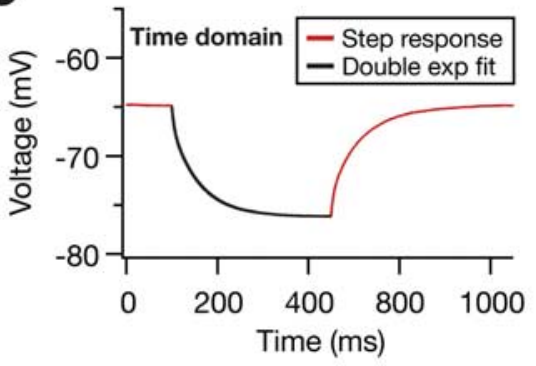

F

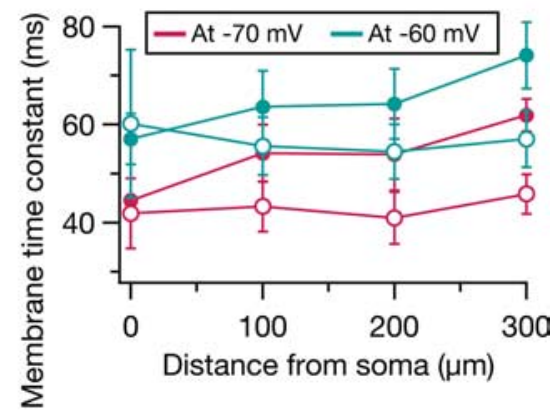

I

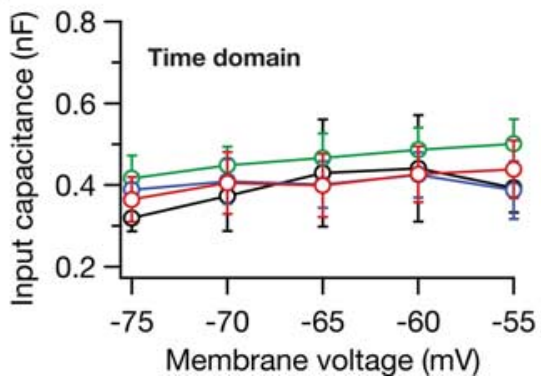

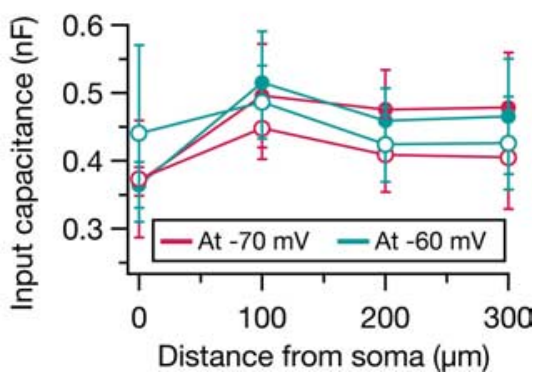

Figure 6. Whole-cell recordings obtained from ZD7288-pretreated slices indicate that membrane time constant and local input capacitance do not significantly change with distance from the soma. A, Schematic of the somatoapical trunk, depicting the experimental design for estimating membrane time constant as a function of distance from the soma. In ZD7288-pretreated slices, voltage responses of the soma and dendrites at various distances (up to $300 \mu \mathrm{m}$ away from the soma) to the Chirp20 stimulus and to a hyperpolarizing current pulse (100 pA) were recorded locally using a whole-cell patch-clamp electrode $\left(V_{m}\right)$. The response to the Chirp20 stimulus and the hyperpolarizing pulse were used to estimate membrane time constant in the frequency domain $(\boldsymbol{B})$ and in the time domain $(\boldsymbol{C}$, respectively. Colors of markers serve as codes for corresponding distances in $\boldsymbol{D}$ and $\boldsymbol{E}$ and $\boldsymbol{G}$ and $\boldsymbol{H}$. $\boldsymbol{B}$, Plot illustrating a Lorentzian fit (black) to the ZAP obtained from pyramidal cell dendrite located at $280 \mu \mathrm{m}$ away (red) from the soma (same dendrite as that shown in Fig. $5 \mathrm{~A}$ ). The fit parameters were impedance amplitude at zero frequency $Z(0) \mid=124.84 \mathrm{M} \Omega$, membrane time constant $\tau=75.03 \mathrm{~ms}$, and local input capacitance $C_{\text {in }}=601 \mathrm{pF}$. C, Plot illustrating a double exponential fit (black) to the ZAP obtained from pyramidal cell dendrite located at $280 \mu$ m away (red) from the soma (same dendrite as in $\boldsymbol{B}$ ). The fit parameters were input resistance $R_{\text {in }}=112.10 \mathrm{M} \Omega$, membrane time constant $\tau=60.65 \mathrm{~ms}$, and local input capacitance $C_{\text {in }}=530 \mathrm{pF}$. The plots of membrane time constant estimated using frequency $(\boldsymbol{D})$ and time $(\boldsymbol{E})$ domain methods as a function of membrane voltage for various distances, and as functions of distance from the soma $(\boldsymbol{F})$ are provided. Similar plots for local input capacitance are also provided $(\boldsymbol{G} \boldsymbol{I})$ in the same order. $\operatorname{In} \boldsymbol{F}$ and $\boldsymbol{I}$, the closed and open circles indicate frequency and time domain estimates, respectively. These plots show that membrane time constant and local input capacitance do not significantly change with distance from the soma, and that the estimates obtained from time and frequency domain methods are not significantly different across various distances from the soma. The number of somatic or dendritic recordings performed to arrive at plots $D-I$ are the same as those shown in Figure 5E. All error bars represent SEMs.

(Fig. $4 F, G$ ), we used two independent methods to estimate this local input capacitance at various locations along the dendritic tree (see Materials and Methods). Recordings obtained from slices pretreated with ZD7288 were used to estimate these parameters, so that the $\mathrm{h}$ conductance does not contribute to the estimates. The frequency domain measures of time constant, input capacitance, and input resistance were similar to their respective time domain counterparts (Fig. 6; supplemental Fig. $5 F-H$, available at www.jneurosci.org as supplemental material). The results also suggested that the time constant (Fig. $6 F$ ), input capacitance (Fig. 6I), and input resistance (supplemental Fig. $5 \mathrm{H}$, available at www.jneurosci.org as supplemental material) did not change sig- nificantly with distance from the soma. Thus, we concluded that distance-dependent increase in the inductive area was attributable to an increase in h-channel density (Magee, 1998; Lorincz et al., 2002) and not to changes in passive properties along the dendritic cable.

The estimates we obtained, and their invariance with distance from the soma are in agreement with previous literature (Golding et al., 2005). In cortical neurons, however, a gradient in input capacitance has been reported, and it has been postulated to play a role in certain functional aspects of the neuron (Williams and Stuart, 2003). Possible reasons behind this discrepancy could stem from differences in the neuronal morphology, especially in 
length (physical and electrotonic), of these two neurons. Furthermore, spine density could also contribute to the measured invariance within the CA1 pyramidal neuron, because the additional spinal surface area would alter the capacitive and resistive properties at a given location (Tsay and Yuste, 2004). It should also be noted that the time constant estimates obtained with either technique are just estimates, given the approximations associated with cable theory (Spruston et al., 1994) and the presence of voltage-gated conductances (Koch, 1984; Koch et al., 1996). For instance, it may be noted from Figure $6 E$, in which time constants are estimated from the response of the neuron to a hyperpolarizing current injection, that the estimate is voltage dependent even with the h conductance blocked. Although the frequency domain estimates for the same set of soma and dendrites are pretty robust across voltages (Fig. 6D), this would imply the presence of other inward rectifying conductances in the membrane that would cause errors in arriving at passive measures (Koch et al., 1996).

\section{LTP is accompanied by spatially widespread changes in intrinsic phase response}

We recently reported that LTP is accompanied by spatially widespread changes in $f_{\mathrm{R}}$ and resonance strength $(Q)$ in CA1 pyramidal neurons (Narayanan and Johnston, 2007). We asked whether this would also be accompanied by an increase in the intrinsic phase response. To test this, we measured changes in $\Phi_{L}$ across the somatoapical trunk using the Chirp15 stimulus, and induced LTP through pairing of antidromic with orthodromic stimulation (Frick et al., 2004; Narayanan and Johnston, 2007). This form of theta burst pairing has been referred to as ATBP and induces robust LTP as measured at various locations along the dendritic tree (Fig. 7B) (Narayanan and Johnston, 2007).

Impedance phase profiles (Fig. $7 E$ ) obtained from responses of a CA1 pyramidal dendrite to the Chirp 15 stimulus before and 40 min after ATBP (Fig. 7C) indicate that both the peak positive phase value and the frequency extent over which positive phase values were obtained increased with ATBP. Quantifying this, we noted that the increases in $\Phi_{L}$ were observed across all three subpopulations (soma, 125 and $250 \mu \mathrm{m}$ ) along the somatoapical trunk (Fig. $7 F, G$ ). Thus, LTP is accompanied by spatially widespread increases in the inductive component of the local input impedance.

\section{Plasticity in intrinsic phase response is attributable to changes in the $h$ current}

Given that the plasticity in inductive phase could occur because of changes in leak conductance, membrane capacitance, or the $h$ conductance (Fig. 3, Table 1), we asked which of these parameters contributed to this impedance plasticity associated with ATBP. To answer this, we first analyzed the RCh circuit to understand the effects of changing each of these parameters on the impedance amplitude profile. We had previously observed that the RC and $\mathrm{Rh}$ circuits act as a low-pass and high-pass filters, respectively, and the convergence of these two filters is what makes an RCh circuit a bandpass filter (Fig. 2C). This observation led us to postulate that a change in $C_{\mathrm{m}}$, in the RCh circuit, should specifically affect the cutoff frequency for the low-pass filter, thus predominantly affecting impedance amplitudes at higher frequencies. Similarly, a change in $\bar{g}_{\mathrm{h}}$ should predominantly affect impedance amplitudes at lower frequencies. We also postulated that change in $R_{\mathrm{m}}$ should affect impedance amplitudes at all frequencies, because $R_{\mathrm{m}}$ changes the cutoff frequencies for both the low-pass and the high-pass filters.

We tested these postulates using simulations on the RCh circuit.
We specifically analyzed the ZAPs for changes in each of these three parameters $\left(R_{\mathrm{m}}, C_{\mathrm{m}}, \bar{g}_{\mathrm{h}}\right)$ toward obtaining an increase in $f_{\mathrm{R}}$ (to replicate what we observe experimentally). These changes (decrease in $R_{\mathrm{m}}$, decrease in $C_{\mathrm{m}}$, or increase in $\bar{g}_{\mathrm{h}}$ ) resulted in the impedance amplitude profiles shown in Figure $8 B$, which confirmed our postulate about the range of frequencies affected by changing each of these parameters (Fig. $8 B, C$ ). We also noted that the impedance phase profile was not a good way to distinguish changes between these three parameters (supplemental Fig. $6 \mathrm{~A}$, available at www.jneurosci.org as supplemental material).

Equipped with this understanding on each of the parameters, we analyzed the ZAPs for the three different locations that we had experimentally recorded from. We observed that, in all three cases, the amount of change at lower frequencies was far greater than that at higher frequencies (Fig. $8 F, G$ ), thus ruling out the role of capacitance in the observed plasticity. Apart from this frequency analysis, we also noted that a reduction in capacitance would imply an increase in $|Z|_{\max }$ (Fig. $8 \mathrm{~B}$, Table 1). Analysis of experimental results suggested that $|Z|_{\max }$ decreased across all three analyzed locations (Fig. $8 H, I$ ), which also rules out the role of capacitance in increasing $f_{\mathrm{R}}$ and $\Phi_{L}$. Although there is a significant reduction in the percentage change in impedance amplitude (Fig. $8 G$ ) with increasing frequency at all three locations, given especially that the $250 \mu \mathrm{m}$ curve does not approach zero at higher frequencies (Fig. $8 F$, green) within the analyzed range of frequencies, we asked whether these changes could be attributable to changes in a leak conductance. A change in a leak conductance to obtain an increase in $f_{\mathrm{R}}$ would mean a reduction in both $Q$ and $\Phi_{L}$ (Table 1), whereas experimentally both $Q$ (Narayanan and Johnston, 2007) and $\Phi_{L}$ (Fig. $7 F$ ) increase across all three locations, thus arguing against the role of a leak conductance. However, changes in properties of $\mathrm{h}$ channels were sufficient to account for experimentally observed changes in all these measurements. We now return to the question of why the reduction in impedance amplitude is higher at higher frequencies for the $250 \mu \mathrm{m}$ population (Fig. $8 \mathrm{~F}$, green). The $\mathrm{h}$ conductance is a typical phenomenological inductance (Figs. 2, 3), in that it acts as an inductance, but also contributes to steady-state input resistance (Mauro et al., 1970; Puil et al., 1988). Thus, the higher density of $\mathrm{h}$ channels in distal dendrites contributes to a greater dependence of input resistance on the $\mathrm{h}$ conductance at distal dendrites than at proximal dendrites or the soma (Magee, 1998; Narayanan and Johnston, 2007). Together, these imply that changes in the h conductance would apparently look like changes in a leak conductance for the $250 \mu \mathrm{m}$ population (Fig. $8 \mathrm{~B}$, red; $F$, green). However, given that changes in h-channel properties were sufficient to account for all experimentally observed changes (especially increases in $Q$ and $\Phi_{L}$ ), these results suggest that plasticity in impedance is attributable to changes in properties of the h channel.

Which of the parameters associated with the h channel contributes to this plasticity: $V_{1 / 2}, \tau_{\mathrm{h}}$, or $\bar{g}_{\mathrm{h}}$ (Table 1 )? To answer this question, we again looked at changes occurring in the ZAP with changes in each of these parameters. We noted that changes in $\tau_{\mathrm{h}}$ do not affect the steady-state input resistance (the impedance amplitude at $f=0$ ) (supplemental Fig. 3B, available at www. jneurosci.org as supplemental material), which is to be expected because the activation/deactivation time constant should not affect the steady-state values of the h conductance or the steadystate input resistance. However, the steady-state input resistance (Narayanan and Johnston, 2007), and the input impedance amplitude closer to zero frequencies (Fig. $8 F$ ) reduced with ATBP across all three locations. Thus, $\tau_{\mathrm{h}}$ can be ruled out as the sole 
A

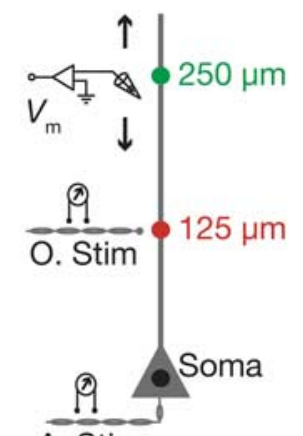

A. Stim
B

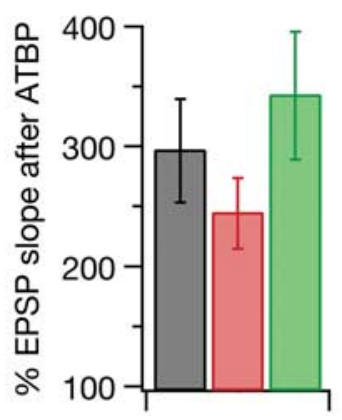

C

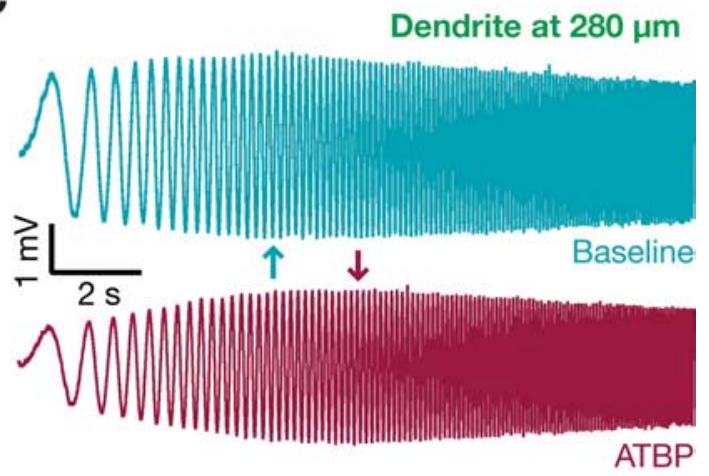

E

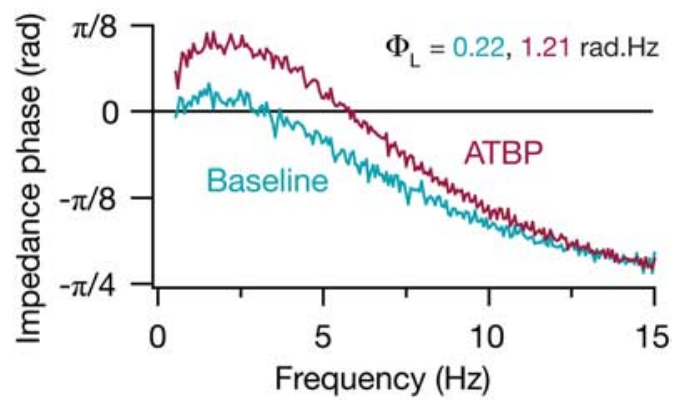

G

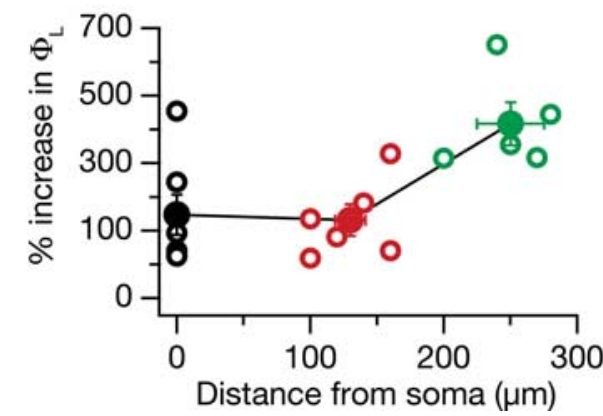

Figure 7. LTP is accompanied by spatially widespread changes in intrinsic phase response. $\boldsymbol{A}$, Schematic of the somatoapical trunk depicting the experimental design for assessing LTP-associated plasticity in inductive phase as a function of distance from the soma. Voltage responses of the soma and dendrites at various distances (up to $\sim 300 \mu \mathrm{m}$ from the soma) were recorded locally using a whole-cell patch-clamp electrode $\left(V_{m}\right)$. Recordings along the somatoapical trunk were binned into three subpopulations (soma, $125 \mu \mathrm{m}$, and $250 \mu \mathrm{m}$ ), depending on the distance of the recording location from the soma. Colors of markers along the somatoapical trunk serve as codes for corresponding distances in $\boldsymbol{B}, \boldsymbol{F}$, and $\boldsymbol{G}$. $\boldsymbol{B}$, Summary plot depicting percentage of EPSP slope measured, for the three subpopulations, at 35-40 min after ATBP, computed with respect to baseline values. $C$, Response of a dendrite, located at $280 \mu \mathrm{m}$ from the soma, to the Chirp15 stimulus during the baseline period and $40 \mathrm{~min}$ after ATBP. The arrows in corresponding colors indicate the location of maximal response in each trace, also illustrating a rightward shift in this location in the ATBP trace with respect to that in the baseline trace. The color code serves as a means to interpret corresponding plots in $\boldsymbol{D}$ and $\boldsymbol{E}$. $\boldsymbol{D}$, Impedance amplitude profile computed from corresponding traces in $\boldsymbol{C}$. The dotted lines indicate the $f_{R}$ and the maximal impedance amplitude $\left(|Z|_{\text {max }}\right)$ for each of the two traces. $E$, Impedance phase profile computed from corresponding traces in $\boldsymbol{C}$. ATBP-induced increases in the amount and the extent of positive phase component may be noted by comparing the ATBP plot to the baseline plot. $\boldsymbol{F}$, Population plots of $\Phi_{L}$ measured before (baseline; open circles) and 40 min after ATBP (ATBP; solid circles) show significant increases in $\Phi_{L}$ (paired Student's $\boldsymbol{t}$ test) after ATBP at all three subpopulations. $\boldsymbol{G}$, Scatterplot of data in $\boldsymbol{F}$. Each open circle represents the percentage of increase, at 35-40 min after ATBP, relative to the respective baseline value of $\Phi_{L}$ in a given experiment. The solid circles represent the average distance and average plasticity for the three populations. The percentage increases in $\Phi_{L}$ were not significantly different across the three populations [Kruskal-Wallis test yielded a significant difference ( $p=0.0326$ ); however, a post hoc Dunn's test did not show significant differences $(p>0.05)$ between any two of the three populations]. All error bars represent SEMs.

parameter that increases $\Phi_{L}$ or $f_{\mathrm{R}}$. However, additional experiments would be required to resolve the ambiguity between $V_{1 / 2}$ and $\bar{g}_{\mathrm{h}}$. Thus, the entire constellation of spatially widespread changes accompanying ATBP [increase in $f_{\mathrm{R}}$, decrease in $R_{\text {in }}$, increase in $Q$ (Narayanan and Johnston, 2007), increase in $\Phi_{L}$ (Fig. $7 F$ ), decrease in $|Z|_{\max }$ (Fig. $8 H$ ), and dominant reductions at lower frequencies (Fig. $8 F, G$ )] was consistent only with changes in $V_{1 / 2}$ and/or $\bar{g}_{\mathrm{h}}$ of the h channel (Table 1 ), and not with changes only in a leak conductance, input capacitance, or $\tau_{\mathrm{h}}$.

\section{Discussion}

The importance of signal phase cannot be overemphasized (Oppenheim and Lim, 1981). For example, it has been demonstrated that the phase at which a hippocampal neuron discharges with respect to its local field potential (LFP) forms a good predictor of the location of the animal within a place field (Jensen and Lisman, 2000; Mehta et al., 2002; O'Keefe and Burgess, 2005). The phase of action potential discharge with respect to the LFP has been suggested to play different roles in the temporal coding of infor- 
A

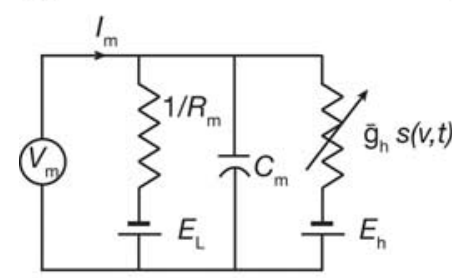

D

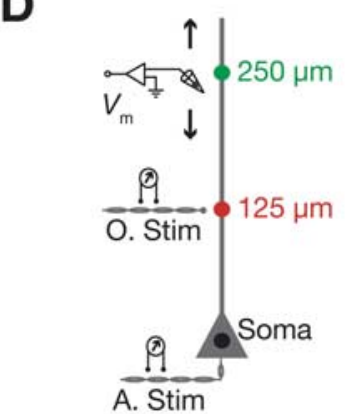

B

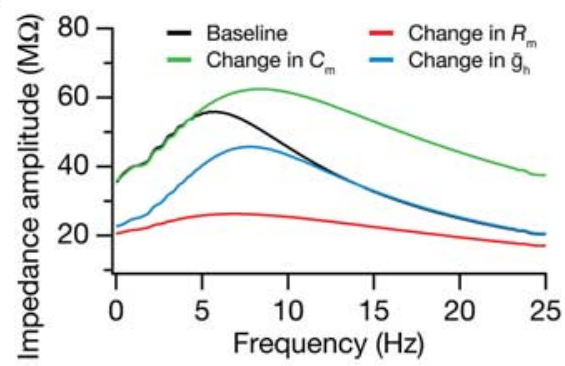

E

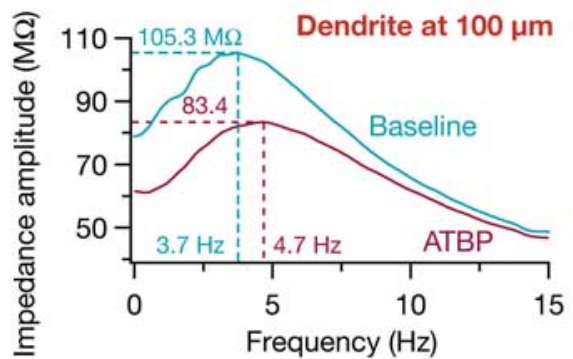

H
C

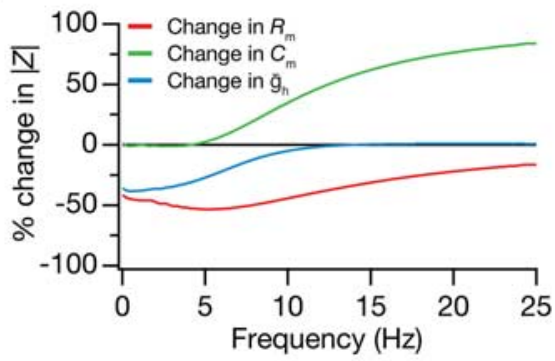

F

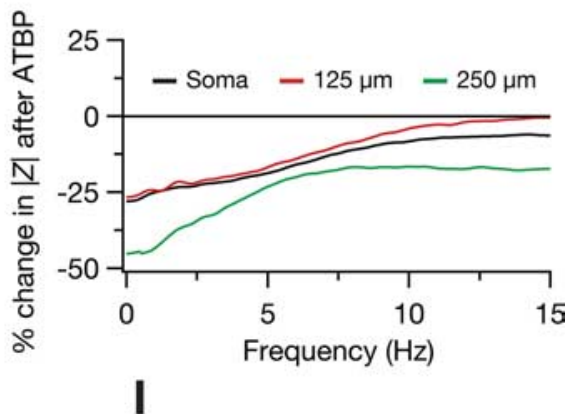

I

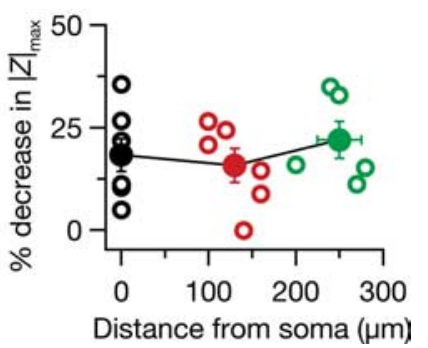

Figure 8. LTP-associated plasticity in impedance properties is attributable to changes in the $h$ current, and not to changes in passive properties. $A$, RCh circuit representing a single compartment neuron model, with the $h$ conductance as the only active mechanism. $B$, Increase in resonance frequency in the model can be obtained by either a reduction in $R_{\mathrm{m}}$ or $C_{\mathrm{m}}$ or an increase in $\bar{g}_{\mathrm{h}}$ (Table 1 ). ZAPs for increasing resonance frequency $\left(f_{\mathrm{R}}\right)$ from a baseline $\left(\right.$ black, $f_{\mathrm{R}}=5.8 \mathrm{~Hz}$; maximal impedance amplitude $|Z|_{\max }=55.9 \mathrm{M} \Omega$ ) through a twofold reduction in $C_{\mathrm{m}}$ (green, $f_{\mathrm{R}}=8.3 \mathrm{~Hz} ;|Z|_{\mathrm{max}}$ $=62.4 \mathrm{M} \Omega$ ), a threefold reduction in $R_{\mathrm{m}}\left(\mathrm{red}, f_{\mathrm{R}}=6.8 \mathrm{~Hz} ;|z|_{\max }=26.2 \mathrm{M} \Omega\right)$, or a twofold increase in $\bar{g}_{\mathrm{h}}\left(\mathrm{blue}, f_{\mathrm{R}}=7.8 \mathrm{~Hz} ;|z|_{\max }=45.7 \mathrm{M} \Omega\right.$ ) are shown. $C$, Percentage changes from the baseline (black in $\boldsymbol{B}$ ) impedance amplitude, $|Z|$, obtained by altering only $R_{\mathrm{m}}$ (red) or $C_{\mathrm{m}}$ (green) or $\bar{g}_{\mathrm{h}}$ (blue) plotted as a function of frequency. $\boldsymbol{D}$, Experimental design for ATBP. Details are the same as Figure 7A. E, ZAPs of a dendrite, located at $100 \mu \mathrm{m}$ from the soma, computed during the baseline period and $40 \mathrm{~min}$ after ATBP. The increase in resonance frequency (numbers close to the $x$-axis) and the reduction in the maximal impedance amplitude (numbers close to the $y$-axis) may be noted. Representative ZAPs before and after ATBP for the somatic population and the $250 \mu \mathrm{m}$ dendritic subpopulations are provided in supplemental Figure $6 B$ (available at www.jneurosci.org as supplemental material) and Figure $7 D$, respectively. $F$, Average percentage change in $|Z|$ obtained for each of the three subpopulations plotted as a function of frequency. This was computed by dividing ZAP obtained after ATBP by the corresponding ZAP during baseline (example shown in $E$ ) for each somatic and dendritic recording, converting the ratio to percentage change, and averaging these percentages over all recordings within the three subpopulations. $\mathbf{G}$, To quantify the average percentage change in $|Z|$ as a function of frequency, we split the analyzed frequency range $(0-15 \mathrm{~Hz})$ to three $5 \mathrm{~Hz}$ groups, and measured the area under the curves (AUCs) shown in $\boldsymbol{E}$ for each of the three subpopulations. For all three subpopulations (soma, $125 \mu \mathrm{m}$, and $250 \mu \mathrm{m}$ ), the amount of changes in higher frequencies was significantly lower than those at lower frequencies. Color-coded asterisk ${ }^{*}$ ) above a group represents that the values within that group were significantly different compared with the values in the $0-5 \mathrm{~Hz}$ group of the same subpopulation; none of the other comparisons was significantly different. Explicitly, within all three subpopulations, the values in the $5-10 \mathrm{~Hz}$ group and the $10-15 \mathrm{~Hz}$ group were significantly less than those in the $0-5 \mathrm{~Hz}$ group (repeated-measures ANOVA, $p<0.0001$, followed by Bonferroni's multiple-comparison test, $p<0.01$ ). In all three subpopulations, the values in the $5-10 \mathrm{~Hz}$ group were not significantly different from those in the $10-15 \mathrm{~Hz}$ range (Bonferroni's multiple-comparison test, $p>0.05$ ). Across subpopulations, within each of the three frequency ranges, none of the values was significantly different from the other (one-way ANOVA, $p>0.05)$. $\boldsymbol{H}$, Population plots of maximal impedance amplitude $\left(|Z|_{\text {max }}\right)$ measured before (baseline; open circles) and 40 min after ATBP (ATBP; solid circles) show significant reductions in $|Z|_{\max }$ (paired Student's $t$ test) after ATBP at all three subpopulations. $\boldsymbol{I}$, Scatterplot of data in $\boldsymbol{H}$. Each open circle represents the percentage of reduction, at $35-40$ min after ATBP, relative to the respective baseline value of $|Z|_{\max }$ in a given experiment. The solid circles represent the average distance and average plasticity for the three subpopulations. The percentage reductions in $|Z|_{\max }$ were not significantly different across the three subpopulations (Kruskal-Wallis test, $p>0.4$ ). All error bars represent SEMs.

mation within the brain (O'Keefe and Recce, 1993; Kamondi et al., 1998; Buzsaki, 2002; Mehta et al., 2002; Hasselmo, 2005; Lengyel et al., 2005; Lisman, 2005; O'Keefe and Burgess, 2005; Siapas et al., 2005; Fries et al., 2007). In this study, we offered direct experimental evidence that the intrinsic phase response of a hippocampal pyramidal neuron undergoes significant changes as a function of distance from the soma. We demonstrated that long-term potentiation is accompanied by spatially widespread changes in this intrinsic phase response of a neuron. We also argued, using two distinct methods of analysis, that changes in $\mathrm{h}$-channel properties, and not those in passive properties, mediate the location and activity dependence of the intrinsic phase response.

\section{$\mathrm{h}$ channel as a location-dependent and plastic inductance}

Our results argue for the $\mathrm{h}$ channel acting as a phenomenological inductive element that is dependent on membrane voltage (Figs. 3, 4), dendritic location (Fig. 4), and activity (Fig. 7). In electrical 
circuits, an inductor acts as a voltage integrator; the current through an inductor is directly proportional to the integral of the voltage across it (Middendorf, 1965; Skilling, 1965). Given that the $h$ conductance behaves as an inverse leaky voltage-dependent inductor (compare Figs. 1, 2), it is not surprising that increase in $\mathrm{h}$ conductance reduces neuronal integration (Magee, 1998). Furthermore, the roles of the $\mathrm{h}$ channel in oscillatory systems (Hutcheon and Yarom, 2000; Robinson and Siegelbaum, 2003) and in bandpass filtering in neurons (Hutcheon and Yarom, 2000; Narayanan and Johnston, 2007) are consistent with well established functions of electrical inductors (Middendorf, 1965; Skilling, 1965).

The gradient and plasticity of h-channel properties in the hippocampal pyramidal cell enable it to act as an adaptive, inputdependent, nonlinear, voltage-dependent bandpass filter (Hutcheon and Yarom, 2000; Narayanan and Johnston, 2007). Because the filter cutoff frequency and the intrinsic phase response are modulated by voltage (Figs. $2-4$ ), the h channel could be considered to be a voltage-controlled inductor, a property that would be common to all resonating conductances. Thus, modulation of any resonating conductance, either through activitydependent plasticity (Fan et al., 2005; Brager and Johnston, 2007) or through neuromodulators (Pape, 1996; Delmas and Brown, 2005), would affect neuronal integration, and alter the intrinsic amplitude and phase responses of the bandpass filter that the conductance mediates.

\section{Phenomenological reactances, timing, and temporal coding} Phenomenological inductances (resonating conductances; e.g., $\mathrm{h}$ and $\mathrm{M}$ conductances) mediate lead in impedance phase, whereas phenomenological capacitances (amplifying conductances; e.g., persistent sodium) would mediate lag in impedance phase at frequency ranges that depend on their kinetics. These reactances have been reported to express subneuronal gradients within various neurons (Magee, 1998; Kole et al., 2006; Hu et al., 2007) and are known to exhibit modulation and activity-dependent plasticity (Pape, 1996; Cantrell and Catterall, 2001; Delmas and Brown, 2005; Fan et al., 2005; Brager and Johnston, 2007). Our results demonstrate that location dependence and plasticity in a phenomenological inductance ( $\mathrm{h}$ conductance) translates to similar characteristics in the intrinsic phase responses of the neuron. Generalizing from these results, we postulate that phenomenological reactances mediate a mechanism that provides independent and adaptive control for each neuron to differentially regulate arrival times of its various inputs. Furthermore, conclusions from this and other related studies (Fan et al., 2005; Brager and Johnston, 2007; Narayanan and Johnston, 2007) would endow single neurons with mechanisms to regulate their excitability, preferred frequencies, and intrinsic phase response, which, through their influences on subthreshold integration could control the timing of action potential generation. Such timing control could play important roles in establishing and maintaining phase locking and synchrony (Buzsaki, 2002; O'Keefe and Burgess, 2005; Siapas et al., 2005), in mediating phase precession (O'Keefe and Recce, 1993; Kamondi et al., 1998; O'Keefe and Burgess, 2005; Burgess et al., 2007; Hasselmo et al., 2007; Maurer and McNaughton, 2007), and in relating neural dynamics to neural coding through phase-resetting curves and spike-timing dependent plasticity (Lengyel et al., 2005; Ermentrout et al., 2007). Finally, phenomenological reactances and their plasticity could provide additional substrates for the postulated roles of oscillations, timing, and temporal coding in the hippocampus (Jensen and Lisman, 2000; Buzsaki, 2002; Mehta et al., 2002; Hasselmo,
2005; O'Keefe and Burgess, 2005), the entorhinal cortex (Burgess et al., 2007; Giocomo et al., 2007; Hasselmo et al., 2007), and in sensory regions in which timing is of paramount importance (Strohmann et al., 1994; Yamoah et al., 1998). Future studies on temporal coding could thus focus on the potential role of voltagegated ion channels in modulating subthreshold temporal responses rather than just mediating spike-timing modulation through changes in excitability.

\section{Impedance is a powerful measure of intrinsic excitability}

It is now widely acknowledged that intrinsic excitability plays an important role in memory encoding and representation (Zhang and Linden, 2003; Frick and Johnston, 2005; Kim and Linden, 2007). Most measures of neuronal excitability rely on subthreshold and suprathreshold DC current injections to the neuron. However, hippocampal neurons reside within an oscillating neuronal network, and in vivo intracellular recordings have shown sinusoidal fluctuations in membrane potentials (Kamondi et al., 1998; Lee et al., 2006). Furthermore, the frequency response properties of these neurons have distinct resonant characteristics (Hu et al., 2002), with the optimal response frequency increasing with distance from the soma (Narayanan and Johnston, 2007). Against the background of these experimental observations, it is clear that oscillatory patterns form natural stimuli to hippocampal pyramidal neurons. Consequently, analyzing intrinsic excitability using sinusoidal current injections would provide a greater understanding of neuronal information processing than those obtained with DC current injections. Furthermore, the dynamical and time-varying nature of the inputs received by neurons would also recommend a frequency-dependent measure of intrinsic excitability as opposed to steady-state measures.

Neuronal impedance forms a powerful and versatile measure of intrinsic excitability, because its magnitude provides a measure of frequency-dependent excitability of neuronal compartments, and its phase indicates the temporal relationship between the input current and output voltage of that compartment at various frequencies (Figs. 2-5). One may compare this with a more commonly used measure of neuronal excitability, its steady-state input resistance, which is just the impedance magnitude at zero frequency, and thus provides neither a broad-spectrum measure of excitability nor the temporal relationship between the input and output. Our study demonstrates the usefulness of impedance in answering a number of physiologically relevant questions. Specifically, we showed that impedance analysis is useful in understanding dependencies of (Figs. 2-5) and plasticity in (Figs. 7, 8) neuronal temporal response properties, estimating time constants (Fig. 6), and discriminating between changes in passive versus active neuronal properties (Figs. 6, 8). Finally, resonance properties and the constellation of measurements that can be obtained from impedance analysis (Table 1) also can provide estimates of parameters related to the underlying phenomenological inductance (Figs. 2-5). For instance, the voltage at which the $f_{\mathrm{R}}$ versus $V_{\mathrm{m}}$ curve peaks can provide a measure of the $V_{1 / 2}$ of the $h$ channel (Brager and Johnston, 2007; Narayanan and Johnston, 2007). Thus, neuronal impedance offers greater insights into the frequency dependence of and temporal interactions among various dendritic compartments within a single neuron, and provides a powerful tool in assessing the roles of neuronal intrinsic dynamics (Marder et al., 1996; Narayanan and Johnston, 2007) and excitability (Zhang and Linden, 2003; Frick and Johnston, 2005; Kim and Linden, 2007) in learning and memory.

In conclusion, we provided direct experimental evidence that 
the $h$ channel contributes a location- and activity-dependent inductive component to the input impedance of CA1 pyramidal soma and dendrites. This demonstration relied on the locationdependent and plastic nature of positive reactance and thus the positive impedance phase offered by such an inductive element. Such location-dependent and plastic intrinsic phase response, in conjunction with similar characteristics of resonance properties and excitability (Fan et al., 2005; Brager and Johnston, 2007; Narayanan and Johnston, 2007), illustrates the important role of the $\mathrm{h}$ channel in regulating dendritic integration and action potential generation in a hippocampal pyramidal neuron. Furthermore, the contribution of dendritic ion channels to intrinsic phase response adds another dimension to the growing body of literature on active dendrites and their plasticity (London and Hausser, 2005; Magee and Johnston, 2005). Future studies could focus on the functional consequences of adaptive inductive elements, especially with respect to dendritic information processing (Kamondi et al., 1998; Magee, 1999, 2003) and temporal coding (Kamondi et al., 1998; Buzsaki, 2002; Mehta et al., 2002; Lengyel et al., 2005; Lisman, 2005; O'Keefe and Burgess, 2005; Siapas et al., 2005; Fries et al., 2007). Finally, although we focused on the hippocampus, our conclusions on various aspects of intrinsic phase responses and on the power of impedance as a measure of intrinsic dynamics and excitability extend to other regions of the brain as well.

\section{References}

Armstrong-Gold CE, Rieke F (2003) Bandpass filtering at the rod to secondorder cell synapse in salamander (Ambystoma tigrinum) retina. J Neurosci 23:3796-3806.

Brager DH, Johnston D (2007) Plasticity of intrinsic excitability during long-term depression is mediated through mGluR-dependent changes in $I_{\mathrm{h}}$ in hippocampal CA1 pyramidal neurons. J Neurosci 27:13926-13937.

Burgess N, Barry C, O'Keefe J (2007) An oscillatory interference model of grid cell firing. Hippocampus 17:801-812.

Buzsaki G (2002) Theta oscillations in the hippocampus. Neuron 33:325-340.

Cantrell AR, Catterall WA (2001) Neuromodulation of $\mathrm{Na}^{+}$channels: an unexpected form of cellular plasticity. Nat Rev Neurosci 2:397-407.

Carnevale NT, Hines ML (2006) The NEURON book. Cambridge, UK: Cambridge UP.

Cole KS (1932) Electrical phase angle of cell membranes. J Gen Physiol 15:641-649.

Cole KS (1941) Rectification and inductance in the squid giant axon. J Gen Physiol 25:29-51.

Cole KS (1949) Some physical aspects of bioelectric phenomena. Proc Natl Acad Sci USA 35:558-566.

Cole KS (1968) Membranes, ions and impulses: a chapter of classical biophysics. Berkeley, CA: University of California Berkeley.

Cole KS, Baker RF (1941) Longitudinal impedance of the squid giant axon. J Gen Physiol 24:771-788.

Delmas P, Brown DA (2005) Pathways modulating neural KCNQ/M (Kv7) potassium channels. Nat Rev Neurosci 6:850-862.

Demontis GC, Longoni B, Barcaro U, Cervetto L (1999) Properties and functional roles of hyperpolarization-gated currents in guinea-pig retinal rods. J Physiol (Lond) 515:813-828.

Erchova I, Kreck G, Heinemann U, Herz AV (2004) Dynamics of rat entorhinal cortex layer II and III cells: characteristics of membrane potential resonance at rest predict oscillation properties near threshold. J Physiol (Lond) 560:89-110.

Ermentrout GB, Galan RF, Urban NN (2007) Relating neural dynamics to neural coding. Phys Rev Lett 99:248103.

Fan Y, Fricker D, Brager DH, Chen X, Lu HC, Chitwood RA, Johnston D (2005) Activity-dependent decrease of excitability in rat hippocampal neurons through increases in $I_{\mathrm{h}}$. Nat Neurosci 8:1542-1551.

Frick A, Johnston D (2005) Plasticity of dendritic excitability. J Neurobiol 64:100-115.

Frick A, Magee J, Johnston D (2004) LTP is accompanied by an enhanced local excitability of pyramidal neuron dendrites. Nat Neurosci 7:126-135.
Fries P, Nikolic D, Singer W (2007) The gamma cycle. Trends Neurosci 30:309-316.

Gasparini S, DiFrancesco D (1997) Action of the hyperpolarizationactivated current $\left(I_{\mathrm{h}}\right)$ blocker ZD 7288 in hippocampal CA1 neurons. Pflügers Arch 435:99-106.

Gasparini S, Migliore M, Magee JC (2004) On the initiation and propagation of dendritic spikes in CAl pyramidal neurons. J Neurosci 24:11046-11056.

Giocomo LM, Zilli EA, Fransen E, Hasselmo ME (2007) Temporal frequency of subthreshold oscillations scales with entorhinal grid cell field spacing. Science 315:1719-1722.

Golding NL, Mickus TJ, Katz Y, Kath WL, Spruston N (2005) Factors mediating powerful voltage attenuation along CA1 pyramidal neuron dendrites. J Physiol (Lond) 568:69-82.

Gutfreund Y, Yarom Y, Segev I (1995) Subthreshold oscillations and resonant frequency in guinea-pig cortical neurons: physiology and modelling. J Physiol (Lond) 483:621-640.

Hasselmo ME (2005) What is the function of hippocampal theta rhythm? Linking behavioral data to phasic properties of field potential and unit recording data. Hippocampus 15:936-949.

Hasselmo ME, Giocomo LM, Zilli EA (2007) Grid cell firing may arise from interference of theta frequency membrane potential oscillations in single neurons. Hippocampus 17:1252-1271.

Hodgkin AL, Huxley AF (1952) A quantitative description of membrane current and its application to conduction and excitation in nerve. J Physiol (Lond) 117:500-544.

Hu H, Vervaeke K, Storm JF (2002) Two forms of electrical resonance at theta frequencies, generated by $\mathrm{M}$-current, $\mathrm{h}$-current and persistent $\mathrm{Na}^{+}$ current in rat hippocampal pyramidal cells. J Physiol (Lond) 545:783-805.

Hu H, Vervaeke K, Storm JF (2007) M-channels (Kv7/KCNQ channels) that regulate synaptic integration, excitability, and spike pattern of CA1 pyramidal cells are located in the perisomatic region. J Neurosci 27:1853-1867.

Hutcheon B, Yarom Y (2000) Resonance, oscillation and the intrinsic frequency preferences of neurons. Trends Neurosci 23:216-222.

Hutcheon B, Miura RM, Puil E (1996a) Subthreshold membrane resonance in neocortical neurons. J Neurophysiol 76:683-697.

Hutcheon B, Miura RM, Puil E (1996b) Models of subthreshold membrane resonance in neocortical neurons. J Neurophysiol 76:698-714.

Jensen O, Lisman JE (2000) Position reconstruction from an ensemble of hippocampal place cells: contribution of theta phase coding. J Neurophysiol 83:2602-2609.

Kamondi A, Acsady L, Wang XJ, Buzsaki G (1998) Theta oscillations in somata and dendrites of hippocampal pyramidal cells in vivo: activitydependent phase-precession of action potentials. Hippocampus 8:244-261.

Kim SJ, Linden DJ (2007) Ubiquitous plasticity and memory storage. Neuron 56:582-592.

Koch C (1984) Cable theory in neurons with active, linearized membranes. Biol Cybern 50:15-33.

Koch C, Rapp M, Segev I (1996) A brief history of time (constants). Cereb Cortex 6:93-101.

Kole MH, Hallermann S, Stuart GJ (2006) Single $I_{\mathrm{h}}$ channels in pyramidal neuron dendrites: properties, distribution, and impact on action potential output. J Neurosci 26:1677-1687.

Lee AK, Manns ID, Sakmann B, Brecht M (2006) Whole-cell recordings in freely moving rats. Neuron 51:399-407.

Lengyel M, Kwag J, Paulsen O, Dayan P (2005) Matching storage and recall: hippocampal spike timing-dependent plasticity and phase response curves. Nat Neurosci 8:1677-1683.

Leung LS, Yu HW (1998) Theta-frequency resonance in hippocampal CA1 neurons in vitro demonstrated by sinusoidal current injection. J Neurophysiol 79:1592-1596.

Lisman J (2005) The theta/gamma discrete phase code occuring during the hippocampal phase precession may be a more general brain coding scheme. Hippocampus 15:913-922.

Llinas RR (1988) The intrinsic electrophysiological properties of mammalian neurons: insights into central nervous system function. Science 242:1654-1664.

London M, Hausser M (2005) Dendritic computation. Annu Rev Neurosci 28:503-532. 
Lorincz A, Notomi T, Tamas G, Shigemoto R, Nusser Z (2002) Polarized and compartment-dependent distribution of HCN1 in pyramidal cell dendrites. Nat Neurosci 5:1185-1193.

Magee JC (1998) Dendritic hyperpolarization-activated currents modify the integrative properties of hippocampal CA1 pyramidal neurons. J Neurosci 18:7613-7624.

Magee JC (1999) Dendritic $I_{\mathrm{h}}$ normalizes temporal summation in hippocampal CA1 neurons. Nat Neurosci 2:848.

Magee JC (2003) A prominent role for intrinsic neuronal properties in temporal coding. Trends Neurosci 26:14-16.

Magee JC, Johnston D (2005) Plasticity of dendritic function. Curr Opin Neurobiol 15:334-342.

Mao BQ, MacLeish PR, Victor JD (2003) Role of hyperpolarizationactivated currents for the intrinsic dynamics of isolated retinal neurons. Biophys J 84:2756-2767.

Marder E, Abbott LF, Turrigiano GG, Liu Z, Golowasch J (1996) Memory from the dynamics of intrinsic membrane currents. Proc Natl Acad Sci USA 93:13481-13486.

Maurer AP, McNaughton BL (2007) Network and intrinsic cellular mechanisms underlying theta phase precession of hippocampal neurons. Trends Neurosci 30:325-333.

Mauro A (1961) Anomalous impedance, a phenomenological property of time-variant resistance. An analytic review. Biophys J 1:353-372.

Mauro A, Conti F, Dodge F, Schor R (1970) Subthreshold behavior and phenomenological impedance of the squid giant axon. J Gen Physiol 55:497-523.

Mehta MR, Lee AK, Wilson MA (2002) Role of experience and oscillations in transforming a rate code into a temporal code. Nature 417:741-746.

Middendorf WH (1965) Introductory network analysis. Boston: Allyn and Bacon.

Narayanan R, Johnston D (2007) Long-term potentiation in rat hippocampal neurons is accompanied by spatially widespread changes in intrinsic oscillatory dynamics and excitability. Neuron 56:1061-1075.

O'Keefe J, Burgess N (2005) Dual phase and rate coding in hippocampal place cells: theoretical significance and relationship to entorhinal grid cells. Hippocampus 15:853-866.

O’Keefe J, Recce ML (1993) Phase relationship between hippocampal place units and the EEG theta rhythm. Hippocampus 3:317-330.

Oppenheim AV, Lim JS (1981) The importance of phase in signals. Proc IEEE 69:529-541.

Pape HC (1996) Queer current and pacemaker: the hyperpolarizationactivated cation current in neurons. Annu Rev Physiol 58:299-327.
Pike FG, Goddard RS, Suckling JM, Ganter P, Kasthuri N, Paulsen O (2000) Distinct frequency preferences of different types of rat hippocampal neurones in response to oscillatory input currents. J Physiol (Lond) 529:205-213.

Puil E, Gimbarzevsky B, Miura RM (1986) Quantification of membrane properties of trigeminal root ganglion neurons in guinea pigs. J Neurophysiol 55:995-1016.

Puil E, Gimbarzevsky B, Spigelman I (1988) Primary involvement of $\mathrm{K}^{+}$ conductance in membrane resonance of trigeminal root ganglion neurons. J Neurophysiol 59:77-89.

Robinson RB, Siegelbaum SA (2003) Hyperpolarization-activated cation currents: from molecules to physiological function. Annu Rev Physiol 65:453-480.

Rumsey CC, Narayanan R, Johnston D (2007) Intraneuronal resonance and frequency response properties of CAl pyramidal neuron models. Soc Neurosci Abstr 33:587.12.

Sabah NH, Leibovic KN (1969) Subthreshold oscillatory responses of the Hodgkin-Huxley cable model for the squid giant axon. Biophys J 9:1206-1222.

Schreiber S, Erchova I, Heinemann U, Herz AV (2004) Subthreshold resonance explains the frequency-dependent integration of periodic as well as random stimuli in the entorhinal cortex. J Neurophysiol 92:408-415.

Siapas AG, Lubenov EV, Wilson MA (2005) Prefrontal phase locking to hippocampal theta oscillations. Neuron 46:141-151.

Skilling HH (1965) Electrical engineering circuits, Ed 2. New York: Wiley.

Spruston N, Jaffe DB, Johnston D (1994) Dendritic attenuation of synaptic potentials and currents: the role of passive membrane properties. Trends Neurosci 17:161-166.

Strohmann B, Schwarz DW, Puil E (1994) Subthreshold frequency selectivity in avian auditory thalamus. J Neurophysiol 71:1361-1372.

Tsay D, Yuste R (2004) On the electrical function of dendritic spines. Trends Neurosci 27:77-83.

Ulrich D (2002) Dendritic resonance in rat neocortical pyramidal cells J Neurophysiol 87:2753-2759.

Williams SR, Stuart GJ (2003) Role of dendritic synapse location in the control of action potential output. Trends Neurosci 26:147-154.

Yamoah EN, Matzel L, Crow T (1998) Expression of different types of inward rectifier currents confers specificity of light and dark responses in type A and B photoreceptors of Hermissenda. J Neurosci 18:6501-6511.

Zhang W, Linden DJ (2003) The other side of the engram: experiencedriven changes in neuronal intrinsic excitability. Nat Rev Neurosci 4:885900 . 